\title{
Self-Adaptive Single-Atom Catalyst Boosting Selective Ferroptosis in Tumor Cells
}

Fangfang Cao, ${ }^{\text {a,e }}$ Yanjuan Sang, ${ }^{\text {a,e }}$ Chaoying Liu, ${ }^{*, b}$ Fuquan Bai, ${ }^{\mathrm{c}}$ Lirong Zheng, ${ }^{\mathrm{d}}$ Jinsong Ren*,a,e and Xiaogang $\mathrm{Qu}^{*, \mathrm{a}, \mathrm{e}}$

${ }^{a}$ State Key Laboratory of Rare Earth Resources Utilization and Laboratory of Chemical Biology, Changchun Institute of Applied Chemistry, Chinese Academy of Sciences, Jilin, 130022, P. R. China

${ }^{\mathrm{b}}$ Department of Respiratory Medicine, First Affiliated Hospital, Jilin University, Jilin, 130021, P. R. China

${ }^{c}$ International Joint Research Laboratory of Nano-Micro Architecture Chemistry, Institute of Theoretical Chemistry Jilin University, Jilin, 130021, P. R. China

${ }^{\mathrm{d}}$ Beijing Synchrotron Radiation Facility, Institute of High Energy Physics, Chinese Academy of Sciences, Beijing 100049, China

${ }^{\mathrm{e}}$ University of Science and Technology of China, Hefei, Anhui 230029, P. R. China

*Email: jren@ ciac.ac.cn, xqu@ @iac.ac.cn 


\section{Experimental Section}

Chemicals. Zinc nitrate hexahydrate $\left(\mathrm{Zn}\left(\mathrm{NO}_{3}\right)_{2} \cdot 6 \mathrm{H}_{2} \mathrm{O}\right)$, iron (II) sulfate heptahydrate $\left(\mathrm{FeSO}_{4} \cdot 7 \mathrm{H}_{2} \mathrm{O}\right)$, methanol, hydrogen peroxide $\left(\mathrm{H}_{2} \mathrm{O}_{2}, 30 \%\right)$, and terephthalic acid (TA) were achieved from Beijing Chemicals (Beijing, China). Iron (III) 2,4-pentanedionate ( $\left.\mathrm{Fe}(\mathrm{acac})_{3}\right)$, 2-methyl imidazole (2-MI), glutathione (GSH), cysteine (Cys), N-Acetyl-L-cysteine (NAC), 1,3-diphenylisobenzofuran (DPBF), deferoxamine mesylate (DFOM), 3-[4,5-dimethylthiazolyl-2-]-2,5-diphenyltetraolium bromide (MTT), propidium iodide (PI) and Calcein AM were ordered from Sigma-Aldrich (St. Louis, MO, USA). 3,3',5,5'-Tetramethylbenzidine (TMB) and L-(+)-ascorbic acid (L-AA) were acquired from Aladdin Reagent (Shanghai, China). Ferrostatin-1 (Fer-1), Z-VAD-FMK, necrostatin-1, liproxstin-1 and vitamine E (VE) were purchased from Shanghai Yuanye Biotechnology Co., Ltd (Shanghai, China). C11-BODIPY ${ }^{581 / 591}$ and singlet oxygen sensor green (SOSG) was purchased from Thermo Fisher (Waltham, MA). GPX4 Rabbit mAb (Catalog No.: A11243), ACSL4 Rabbit mAb (Catalog No.: A20414) and Caspase 3 Rabbit pAb (Catalog No.: A0214) were ordered from ABclonal. Lyso-Tracker Red, dihydroethidiun, 1,3-diphenylisobenzofuran, GSH and GSSG assay kit, cellular glutathione peroxidase assay kit, reactive oxygen species assay kit, BCA protein assay kit, $\beta$-actin antibody, Ferritin rabbit monoclonal antibody and One Step TUNEL Apoptosis Assay Kit were purchased from Beyotime Biotechnology (Shanghai, China). All the reagents were used without further purification. Ultrapure water (18.2 MU; Millpore Co., USA) was used throughout the experiment. DNA oligonucleotides were synthesized by Shanghai Sangon Biological Engineering Technology \& Services (Shanghai, China). The sequences were as follows: MUC-1 aptamer (mDNA): 5'-GGC TAT AGC ACA TGG GTA AAA CGA C-3'. ATP aptamer (aDNA): 5'-AAC CTG GGG GAG TAT TGC GGA GGA AGG T-3'. C-rich ssDNA (cDNA): 5'-CCC TAA CCC TAA CCC TAA CCC T-3'. FAM-labeled mDNA (FAM-mDNA): 5'-FAM/GGC TAT AGC ACA TGG GTA AAA CGA C-3'. macDNA: the mixture of equal mDNA, aDNA and cDNA.

Instruments. Scanning electron microscope (SEM) images were recorded on a Hitachi S-4800 FESEM at working voltage of $10 \mathrm{kV}$ and working current of $10 \mathrm{~A}$. Transmission electron microscope (TEM) images were obtained on a TECNAI G2 equipped with energy dispersive 
spectroscopic (EDS) at $200 \mathrm{kV}$. Fe/PMCS were imaged with high-angle annular dark-field scanning transmission electron microscopy (HAADF-STEM). The HAADF-STEM images were acquired with a Titan 80-300 scanning/transmission electron microscope operated at $300 \mathrm{kV}$, equipped with a probe spherical aberration corrector. X-ray diffractometer (XRD) measurements were performed on a Bruker D8 FOCUS using $\mathrm{Cu}$ K $\alpha$ radiation. Raman spectra were collected on a Renishaw 2000 model confocal microscopy equipped with a CCD detector and a holographic notch filter (Renishiaw Ltd., Gloucestershire, U.K.). X-ray photon spectroscopy (XPS) data were acquired with an ESCALab220i-XL electron spectrometer using $300 \mathrm{~W}$ Al Ka radiation (VG Co., United Kingdom). The content of Fe was determined on the Inductively Coupled Plasma-Optical Emission Spectrometry (ICP-OES, X Series 2, Thermo Scientific, USA). The UV-Vis absorption spectra were recorded using a JASCO V550 UV-Visible spectrophotometer. Fluorescence measurements were carried out on a JASCO FP-6500 spectrofluorometer. The concentration of DNA was measured through the absorbance at $260 \mathrm{~nm}$ at high temperature $\left(95^{\circ} \mathrm{C}\right)$ using a Carry $300 \mathrm{UV} /$ Vis spectrophotometer connected to a thermal peltier controller. The extinction coefficient was calculated from mononucleotide and dinucleotide data through the nearest-neighbor approximation. Melting experiments were conducted on a Cary 300 UV/Vis spectrophotometer equipped with a Peltier temperature control accessory at a heating rate of $1{ }^{\circ} \mathrm{C} \min ^{-1}$. CD spectra experiments were measured on a JASCO J-810 spectropolarimeter equipped with a temperature-controlled water bath. The optical chamber of the CD spectrometer was deoxygenated with dry purified nitrogen (99.99\%) for $5 \mathrm{~min}$ at a speed of $5 \mathrm{~L} \mathrm{~min}^{-1}$ before use and the nitrogen atmosphere kept during experiments. Three scans were accumulated and automatically averaged. The flow cytometry data were recorded using BD LSRFortessaTM cell analyzer. All the photos were taken by a Canon camera.

X-ray Absorption (XAS) measurements and Analyses. The X-ray absorption find structure spectra (Fe K-edge) were collected at 1W1B station in Beijing Synchrotron Radiation Facility (BSRF). The storage rings of BSRF were operated at $2.5 \mathrm{GeV}$ with a maximum current of $250 \mathrm{~mA}$. Using Si (111) double-crystal monochromator, the data collection was carried out in transmission mode using ionization chamber. All spectra were collected in ambient conditions. 
The acquired EXAFS data were processed according to the standard procedures using the ATHENA module implemented in the IFEFFIT software packages. The k3-weighted EXAFS spectra were obtained by subtracting the post-edge background from the overall absorption and then normalizing with respect to the edge-jump step. Subsequently, k3-weighted $\chi(\mathrm{k})$ data of $\mathrm{Fe}$ K-edge were Fourier transformed to real $(\mathrm{R})$ space using a hanning windows $\left(\mathrm{dk}=1.0 \AA^{-1}\right)$ to separate the EXAFS contributions from different coordination shells. To obtain the quantitative structural parameters around central atoms, least-squares curve parameter fitting was performed using the ARTEMIS module of IFEFFIT software packages.

Cell culture. Human breast cancer MCF-7 cell, colorectal cancer CT26 cell, 4T1 murine breast cancer cells, human lung adenocarcinoma A549 cell, human hepatocellular carcinoma HepG2 cells, human cervical adenocarcinoma epithelial HeLa cells, murine RAW264.7 cell and human umbilic vein endothelial cell (HUVEC), were supplied by American Type Culture Collection (ATCC) and cultured with regular growth medium consisting of high glucose DMEM. The cell growth media were supplemented with $10 \%$ heat-inactivated FBS in an atmosphere of $5 \% \mathrm{CO}_{2}$ at $37{ }^{\circ} \mathrm{C}$. The medium was replaced every 3 days, and the cells were digested by trypsin and resuspended in fresh medium before plating.

In vitro cytotoxicity evaluation toward cancer cells. The MCF-7 cells, CT26 cells, $4 \mathrm{~T} 1$ cells, A549 cells, HepG2 cells, and HeLa cells were seeded in 96-well plates at a density of 5000 cells/well for $12 \mathrm{~h}$. Then, macDNA-Fe/PMCS, at indicated concentrations $(0,12.5,25,50,100$, $200 \mu \mathrm{g} / \mathrm{mL}$ ), were added to the cell culture medium and incubated for another $24 \mathrm{~h}$. Meanwhile, to investigate the cytotoxicity of cancer cells under tumor microenvironment, macDNA-Fe/PMCS were also co-incubated with cancer cells at pH 6.5 in the presence of $100 \mu \mathrm{M} \mathrm{H}_{2} \mathrm{O}_{2}$. Cells were incubated for another $24 \mathrm{~h}$. After that, the medium was removed, and the cells were washed with PBS 3 times for MTT assay. Briefly, MTT reagent was added and followed by the incubation at $37{ }^{\circ} \mathrm{C}$ for another $4 \mathrm{~h}$. Then, the medium of each well was replaced by $100 \mu \mathrm{L}$ DMSO and the plate was gently swirled for $2 \mathrm{~min}$ at room temperature at dark to dissolve all formed precipitate. Absorbance values were determined with Bio-Rad model-680 microplate reader at $490 \mathrm{~nm}$ (corrected for background absorbance at $630 \mathrm{~nm}$ ). The cell viability was estimated according to the following equation: Cell Viability $(\%)=\left(\mathrm{OD}_{\text {treated }} / \mathrm{OD}_{\text {control }}\right) \times 100 \% . \mathrm{OD}_{\text {control }}$ was obtained 
in the absence of samples, whereas $\mathrm{OD}_{\text {treated }}$ obtained in the presence of samples.

In vitro cytotoxicity evaluation toward normal cells. The RAW264.7 cells and HUVEC cells were seeded in 96-well plates at a density of 5000 cells/well for $12 \mathrm{~h}$. Then, macDNA-Fe/PMCS, at indicated concentrations $(0,12.5,25,50,100,200 \mu \mathrm{g} / \mathrm{mL})$, were added to the cell culture medium. Cells were incubated for another $24 \mathrm{~h}$. After that, the in vitro cytotoxicity was analyzed by MTT assay.

To evaluate the influence of $\mathrm{H}_{2} \mathrm{O}_{2}$ and $\mathrm{pH}$ on cytotoxicity toward normal cells, RAW264.7 cells, HEK-293T cells and HUVEC cells were seeded in 96-well plates at a density of 5000 cells/well for $24 \mathrm{~h}$. Then, macDNA-Fe/PMCS $(0,12.5,25,50,100,200 \mu \mathrm{g} / \mathrm{mL})$ with or without $100 \mu \mathrm{M}$ $\mathrm{H}_{2} \mathrm{O}_{2}$ were added into each well and co-cultured with cells for $24 \mathrm{~h}$ at different $\mathrm{pH}$ (7.4 or 6.5). Afterwards, they were assessed by MTT assay.

Lysosomal localization of macDNA-Fe/PMCS. MCF-7 or CT26 cells were seeded on 24-well culture plates and grown for $12 \mathrm{~h}$. Then, the cell medium was removed, washed, and $0.5 \mathrm{~mL}$ fresh cell medium containing different concentration of FAM-labeled macDNA-Fe/PMCS was added to the wells and incubated $4 \mathrm{~h}$. After that, the medium was washed with PBS and cells were stained by $50 \mathrm{nM}$ Lyso-Tracker Red. After staining for $30 \mathrm{~min}$ at $37^{\circ} \mathrm{C}$, the cells were washed with PBS and imaged using fluorescence microscopy. The pictures were taken with an Olympus digital camera.

Live/Dead Assay. MCF-7 or CT26 cells were cultured in a 6-well plate for $12 \mathrm{~h}$ and then washed twice by PBS. $100 \mu \mathrm{g} / \mathrm{mL}$ macDNA-Fe/PMCS were added to above culture medium with or without $100 \mu \mathrm{M} \mathrm{H}_{2} \mathrm{O}_{2}$ and incubated at $37{ }^{\circ} \mathrm{C}$ under $5 \% \mathrm{CO}_{2}$ for $24 \mathrm{~h}$. Afterwards, they were stained with calcein AM and PI for $1 \mathrm{~h}$ to investigate their cytotoxicity.

Animal Experiments. Animal experiments were in accordance with the direction of the Regional Ethics Committee for Animal Experiments. All study protocols involving animals were approved by the Jilin University Animal Care and Use Committee. Healthy female Balb/c mice ( 25 g) and BALB/c nude mice ( $20 \mathrm{~g})$ were acquired from the Laboratory Animal Center of Jilin University (Changchun, China). 
Hemolysis test of macDNA-Fe/PMCS. $1 \mathrm{~mL}$ whole blood was collected in tubes containing Li-heparin from the eyes of mouse. $1 \mathrm{~mL}$ blood was mixed with proper amount of $0.9 \%$ saline and centrifuged at $2500 \mathrm{rpm}$ for $5 \mathrm{~min}$. Then, the supernatant was removed, $2 \mathrm{~mL} 0.9 \%$ saline was added again and the mixture was centrifuged at $2500 \mathrm{rpm}$ for $5 \mathrm{~min}$. The procedure was repeated 3-4 times until the supernatant becoming colorless transparent. The precipitated erythrocytes were then dispersed in $0.9 \%$ saline to get erythrocyte suspension. macDNA-Fe/PMCS was added to the erythrocyte suspension to a final concentration of $(12.5,25,50,100,200 \mu \mathrm{g} / \mathrm{mL}$, in tubes numbered as 1-5). Tube 6 (negative control) was placed with erythrocyte suspension diluted with $0.9 \%$ saline, while tube 7 (positive control) was placed with erythrocyte suspension diluted with ultrapure water. The tubes were gently mixed in a rotary shaker, incubated for $4 \mathrm{~h}$ at room temperature and then the hemolysis phenomenon was observed and recorded. The specimens were then centrifuged under $13000 \mathrm{rpm}$ to remove macDNA-Fe/PMCS and the supernatant was analyzed for the presence of the hemoglobin by specific $541 \mathrm{~nm}$ spectrophotometric absorption. Calculation the hemolysis rate $(H R \%)$ by the following equation.

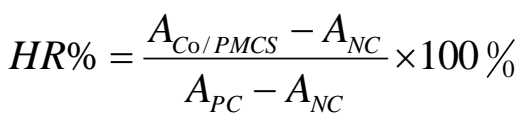

Where $A_{C o / P M C S}, A_{P C}$ and $A_{N C}$ are the absorbance of the sample, the positive control and the negative control, respectively.

In vivo biocompatibility of macDNA-Fe/PMCS. 10 healthy mice were randomly divided into two groups, including saline (control) group and macDNA-Fe/PMCS group. $200 \mu \mathrm{L}$ saline and macDNA-Fe/PMCS $(500 \mu \mathrm{g} / \mathrm{mL})$ were intravenously administrated into mice of the corresponding groups, respectively. The mice were observed for 28 days to evaluate the in vivo biosafety. At the indicated time, the mice were euthanized and their blood samples were collected to perform complete blood panel analysis and serum biochemistry assay at the Jilin ADICON Clinical Laboratories, Inc. The major organs containing heart, liver, spleen, lung, kidney, inestines and stomach were harvested, fixed in $10 \%$ paraformaldehyde, processed into paraffin, sectioned at $\sim 4 \mu \mathrm{m}$, and stained with hematoxylin and eosin (H\&E).

Tumor models. Colon tumor-bearing mice and MCF-7 tumor-bearing mice (tumor size $\approx 100 \mathrm{~mm}^{3}$ ) were used to evaluate the in vivo tumoricidal potentials. The Balb/c mice or nude Balb/c mice 
were subcutaneously inoculated in the oxter region with $1 \times 10^{6}$ CT26 or MCF-7, respectively. They were further raised until the tumors grew to $\sim 100 \mathrm{~mm}^{3}$ before experiment.

In vivo biodistirbution and pharmacokinetic of macDNA-Fe/PMCS. In the quantitative biodistribution analysis, health mice were intravenously injected with $200 \mu \mathrm{L}$ macDNA-Fe/PMCS $(500 \mu \mathrm{g} / \mathrm{mL})$ and randomly divided into 3 groups $(n=5)$. At $4 \mathrm{~h}, 12 \mathrm{~h}$, and $24 \mathrm{~h}$ post administration, the mice were sacrificed, main organs containing heart, liver, spleen, lung, kidney and stomach were excised and weighted, and then digested in aqua regia under heat treatment $\left(80{ }^{\circ} \mathrm{C}\right)$ for 3 days to analyze the content of $\mathrm{Fe}$ in the samples using ICP-OES.

For in vivo pharmacokinetic evaluation, mice $(n=5)$ were intravenously injected with $300 \mu \mathrm{L}$ macDNA-Fe/PMCS (1000 $\mu \mathrm{g} / \mathrm{mL})$. At 5, 15, $30 \mathrm{~min}, 1,2,4,8,12$ and $24 \mathrm{~h}$ post injection, $10 \mu \mathrm{L}$ blood were collected by puncturing the tail vein and dispersed into $990 \mu \mathrm{L}$ aqua regia to dissolve the remained macDNA-Fe/PMCS. The concentrations of Fe were quantified by ICP-OES.

Computational methods. All calculations were performed using density functional theory, as implemented in the Vienna ab initio Simulation Package (VASP) ${ }^{1}$ with the projector augmented wave (PAW) method ${ }^{2}$. used to describe the interaction between core electrons and valence electrons. The exchange and correlation potential were described with the Perdew-Burke-Ernzerhof $(\mathrm{PBE})^{3}$ of the generalized gradient approximation (GGA) ${ }^{4,5}$. The energy cutoff is set to be $500 \mathrm{eV}$ and vacuum region is set to about $15 \AA$. Strong coulomb interactions of $3 \mathrm{~d}$ electron for $\mathrm{Fe}$ atom were described by the DFT+U method. ${ }^{6,7}$ In addition, Grimme-D2 dispersion correction term was chosen to account for the van-der-Waals interactions ${ }^{5,8}$. The k-point sampling of the Brillouin zone was obtained using a $2 \times 2 \times 1$ grid and, both lattice constants along the periodic direction and atomic positions are fully relaxed until the convergence criteria of energy and force is less than $10^{-5} \mathrm{eV}$ and $0.01 \mathrm{eV} / \AA$, respectively. The difference in electronic energy before and after hydrogenation extracted directly from first-principles calculations and $\Delta E_{\mathrm{ZPE}}$ and $\Delta S$ are the zero-point energy correction and entropy change between the absorbed state and gas phase obtained from frequency calculations at $300 \mathrm{~K}$, respectively. A Gaussian smearing of $0.05 \mathrm{eV}$ to the orbital occupation is applied during the geometry optimization and for the total energy computations.

To module the Fe/PMCS SAzymes, a Fe- $\mathrm{N}_{4}$ site is embedded in a periodic $4 \times 4$ graphene 
support ( 32 carbon sites) with lattice parameters $\mathrm{a}=\mathrm{b}=9.84 \AA$ and $\gamma=120^{\circ}$. The vacuum spacing is set to more than $15 \AA$ along the surface normal to avoid the interactions between images. The k-space integration was sampled using a $2 \times 2 \times 1$ Monkhorst-Pack grid. In all the calculations, the spin is unrestricted and initial spin for each $\mathrm{Fe}$ atom is set as 4 . The calculated spin moment of single $\mathrm{Fe}$ atom in $\mathrm{Fe}-\mathrm{N}_{4} / \mathrm{C}$ is $2.07 \mathrm{~B}$, well consistent with the observed magnetic moment of $\sim 2 \mathrm{~B}$. The binding energy was calculated by subtracting the energies of the isolated adsorbate and the catalyst from the total energy of the adsorbed system.

In this study, oxidase-like and peroxidase-like activity on Fe/PMCS was investigated. The oxidase-like reaction mechanism in the free energy profiles calculation are as follows:

(i) $* \mathrm{O}_{2}+\mathrm{H}^{+} \rightarrow * \mathrm{OOH}$

(ii) $* \mathrm{OOH}+\mathrm{H}^{+} \rightarrow * \mathrm{O}+\mathrm{H}_{2} \mathrm{O}$

(iii) $* \mathrm{O}+\mathrm{H}^{+}+\mathrm{TMB} \rightarrow * \mathrm{OH}+$ oxTMB

(iv) $* \mathrm{OH}+\mathrm{H}^{+}+\mathrm{TMB} \rightarrow *+\mathrm{H}_{2} \mathrm{O}+\mathrm{oxTMB}$

The peroxidase-like reaction mechanism along with the following fie elementary steps were considered for Fe/PMCS SAzymes.

(i) $\mathrm{H}_{2} \mathrm{O}_{2}$ (aq) $\rightarrow{ }^{*} \mathrm{H}_{2} \mathrm{O}_{2}+\mathrm{H}_{2} \mathrm{O}$

(ii) $* \mathrm{H}_{2} \mathrm{O}_{2} \rightarrow * \mathrm{O}+\mathrm{H}_{2} \mathrm{O}$

(iii) $* \mathrm{O}+\mathrm{TMB} \rightarrow * \mathrm{OH}+\mathrm{oxTMB}$

(iv) $* \mathrm{OH}+\mathrm{TMB} \rightarrow *+$ oxTMB

The asterisk $(*)$ indicates the adsorption sites on a metal surface. The free energy was calculated by the equation $\mathrm{G}=\mathrm{E}+\mathrm{ZPE}-\mathrm{TS}$, where $\mathrm{E}$ is the total energy, $\mathrm{ZPE}$ is the zero-point energy, $\mathrm{T}$ is the temperature in kelvin, and $\mathrm{S}$ is the entropy. 


\section{Additional Figures and References}
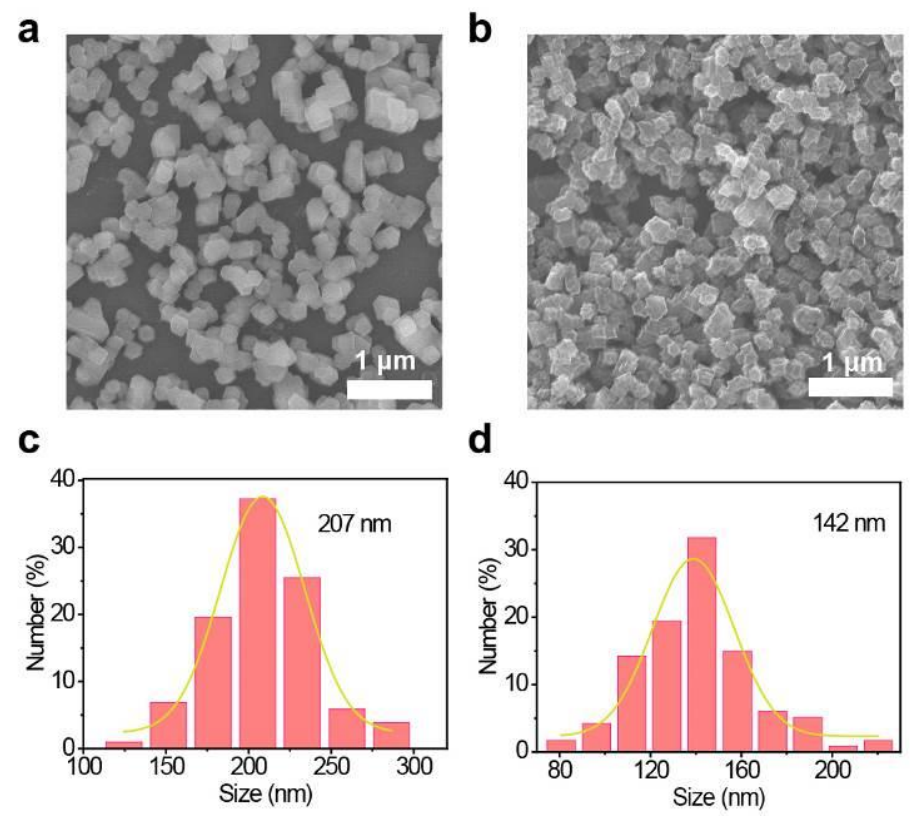

Figure 1. SEM images of a) Fe@ZIF-8 and b) Fe/PMCS. Size distribution analysis of c) Fe@ZIF-8 and d) Fe/PMCS.

SEM images revealed that Fe/PMCS retained the initial rhombic dodecahedral shape and their average sizes shrank to $142 \mathrm{~nm}$ from $207 \mathrm{~nm}$ due to the high-temperature calcination. 

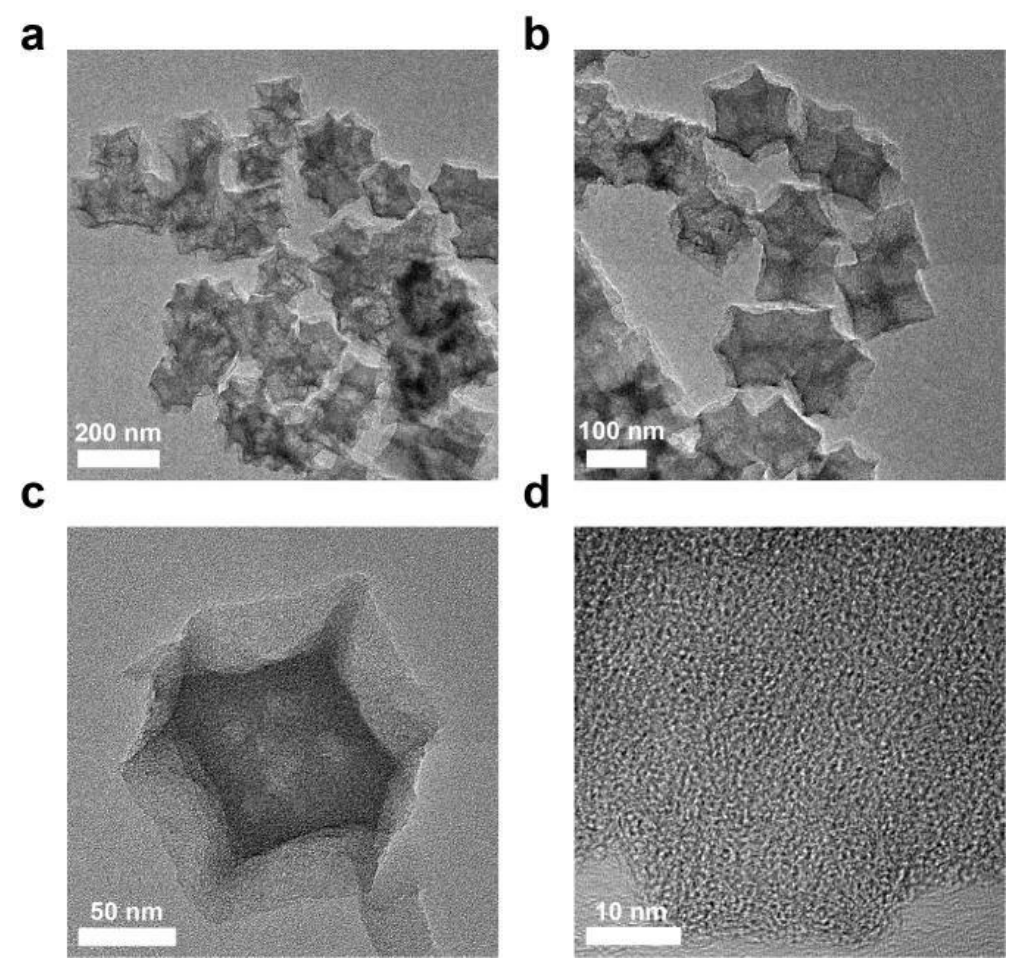

Figure 2. a-c) TEM images and d) HR-TEM image of Fe/PMCS under different magnifications. Notably, no nanoparticles can be found, suggesting that $\mathrm{Fe}$ atoms might be confined in the carbon layers in an atomically dispersed form. 


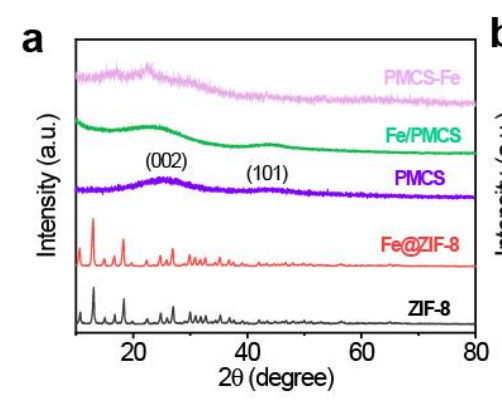

d
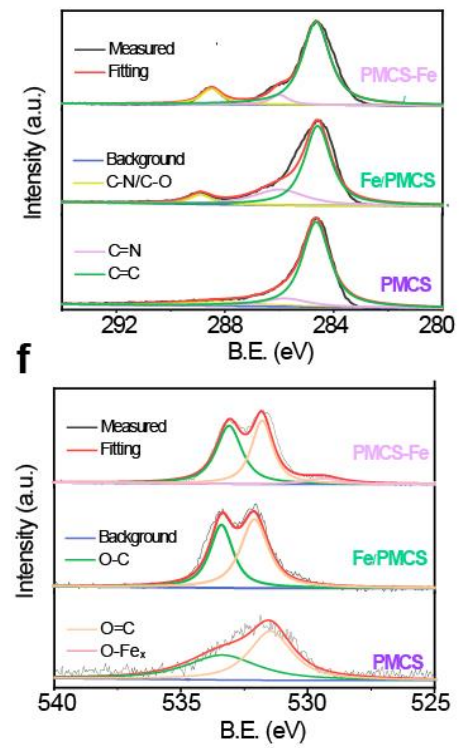
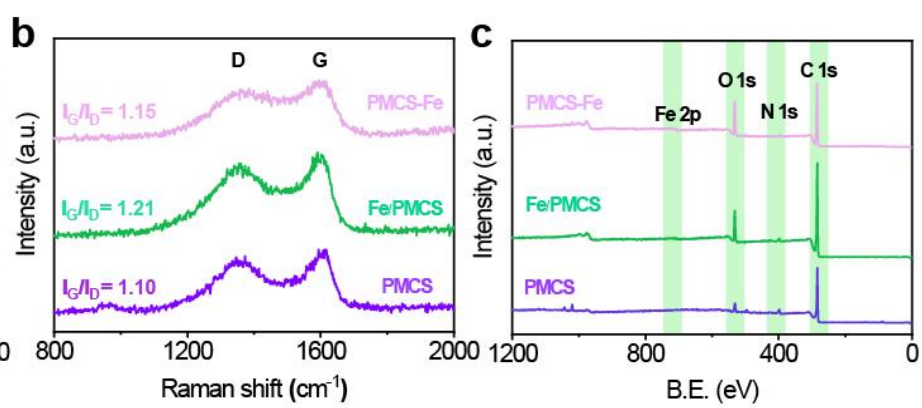

e

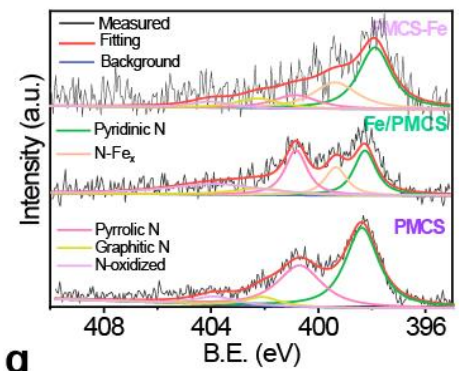

g

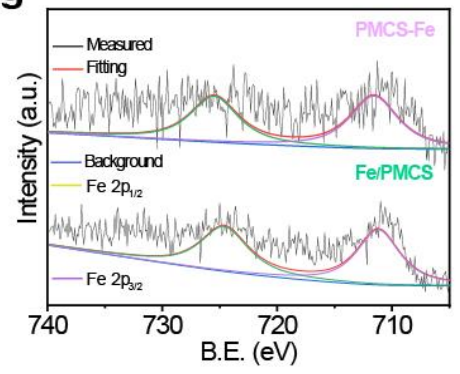

Figure 3. a) Powder XRD patterns of ZIF-8, Fe@ZIF-8, PMCS, Fe/PMCS and PMCS-Fe, respectively. b) Raman spectra, c) XPS survey spectrum, deconvoluted d) C 1s, e) N 1s and f) O 1s XPS spectra of PMCS, Fe/PMCS and PMCS-Fe. g) deconvoluted Fe 2p XPS spectra of Fe/PMCS and PMCS-Fe.

Powder XRD pattern of Fe@ZIF-8 was well matched with that of ZIF-8, suggesting that the encapsulation of Fe did not influence the crystalline of ZIF-8. Fe/PMCS demonstrated a broad shoulder peak in the range $20-30^{\circ}(2 \theta)$, which was in accordance with that of PMCS. This peak was assigned to the (002) plane of the graphitic carbon, as further confirmed by $\mathrm{I}_{\mathrm{G}} / \mathrm{I}_{\mathrm{D}}$ value in Raman spectra. The Raman spectrum showed two main bands located at 1591 and $1352 \mathrm{~cm}^{-1}$ that correspond to the vibration of $\mathrm{sp}^{2}$-bonded carbon atoms and dispersive defect-induced vibrations, respectively. XPS analysis revealed the binding states of $\mathrm{C}, \mathrm{N}$ and $\mathrm{Fe}$ in these samples. High-resolution C 1s spectrum could be fitted into three characteristic peaks, C-N/C-O $(288.9 \mathrm{eV})$, $\mathrm{C}=\mathrm{N}(286 \mathrm{eV})$ and $\mathrm{C}=\mathrm{C}(284.6 \mathrm{eV})$. High-resolution $\mathrm{N}$ 1s spectrum could be fitted into five characteristic peaks, pyridinic $\mathrm{N}(398.2 \mathrm{eV}), \mathrm{N}-\mathrm{Fe}_{\mathrm{x}}(399.3 \mathrm{eV})$, pyrrolic $\mathrm{N}(400.8 \mathrm{eV})$, graphitic $\mathrm{N}$ 
(402.2 eV), and oxidized N (403.9 eV), in which the Fe-Nx peak suggested the good coordination of Fe atom and $\mathrm{N}$. The high-resolution Fe $2 \mathrm{p}$ spectrum with two relatively weak peaks around $711.5 \mathrm{eV}\left(\mathrm{Fe} 2 \mathrm{p}_{3 / 2}\right)$ and $725.5 \mathrm{eV}\left(\mathrm{Fe} 2 \mathrm{p}_{1 / 2}\right)$ demonstrated partially oxidized Fe species in Fe/PMCS, further supporting the existence of Fe-Nx species. High-resolution O 1s spectrum could be fitted into three characteristic peaks, O-C (533.4 eV), $\mathrm{O}=\mathrm{C}(532.1 \mathrm{eV})$ and $\mathrm{O}-\mathrm{Fe}(529.4 \mathrm{eV})$. Obviously, The O-Fe peak only existed in PMCS-Fe, excluding the coordination of Fe atom and O in Fe/PMCS. Thus, the high-resolution $\mathrm{N}$ 1s and $\mathrm{O}$ 1s XPS analysis as well as the studies of XANES and EXAFS demonstrated the accurate structure of Fe atoms (FeN4) in Fe/PMCS. In addition, the peaks around $1000 \mathrm{eV}$ should be identified to $\mathrm{Zn} \mathrm{3p}$, which was consistent to the previous studies. Calcination couldn't remove $\mathrm{Zn}$ completely from PMCS. Although the Zn element was residual in Fe/PMCS, it would not influence the the mechanism studies. Because $\mathrm{Zn}$ element could also be observed in the control samples of PMCS and PMCS-Fe. What's more, as shown in Supplementary Fig.11 and 17, the enzyme-like activity of Fe/PMCS was much higher than that of PMCS and PMCS-Fe, indicating that the ultrahigh activity of Fe/PMCS was derived from Fe. Thus, in the mechanism studies, Fe-N4 model was only established to investigate the catalytic reaction, according to reported works. ${ }^{9-11}$ 


\section{a}

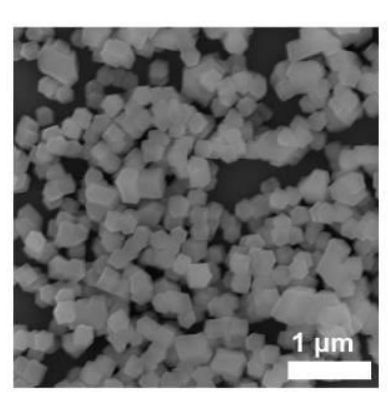

c

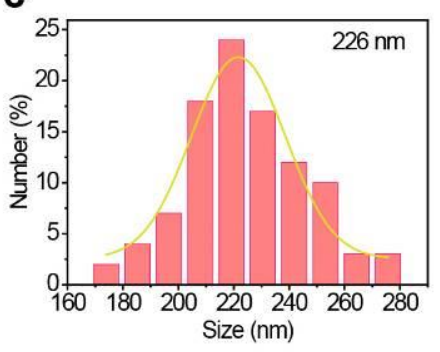

b

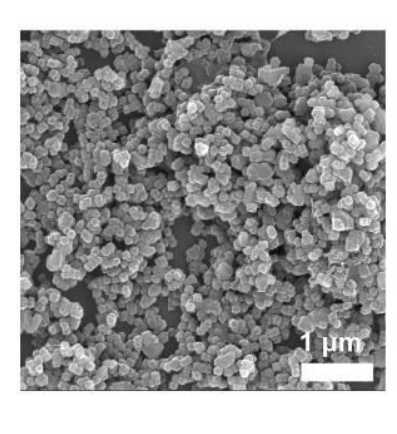

d

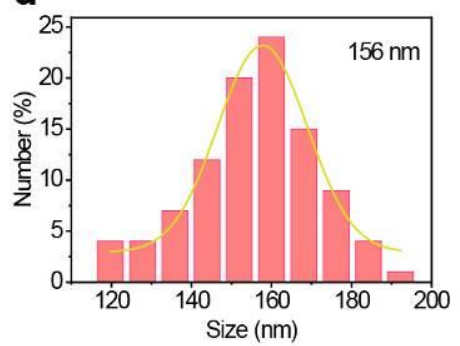

Figure 4. SEM images of a) ZIF-8 and b) PMCS. Size distribution analysis of c) ZIF-8 and d) PMCS. SEM images revealed that Fe/PMCS retained the initial rhombic dodecahedral shape and their average sizes shrank to $156 \mathrm{~nm}$ from $226 \mathrm{~nm}$ due to the thermolysis. 

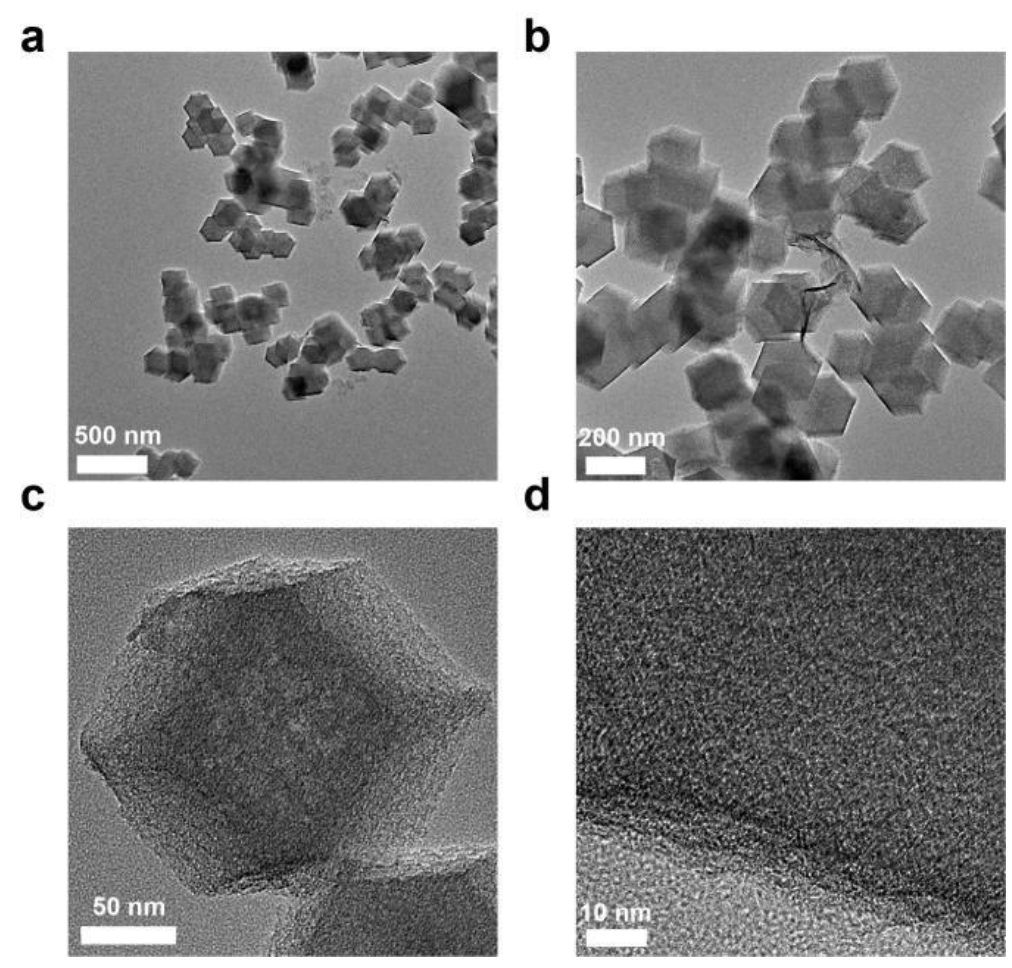

Figure 5. a-c) TEM images and d) HR-TEM image of PMCS under different magnifications. 

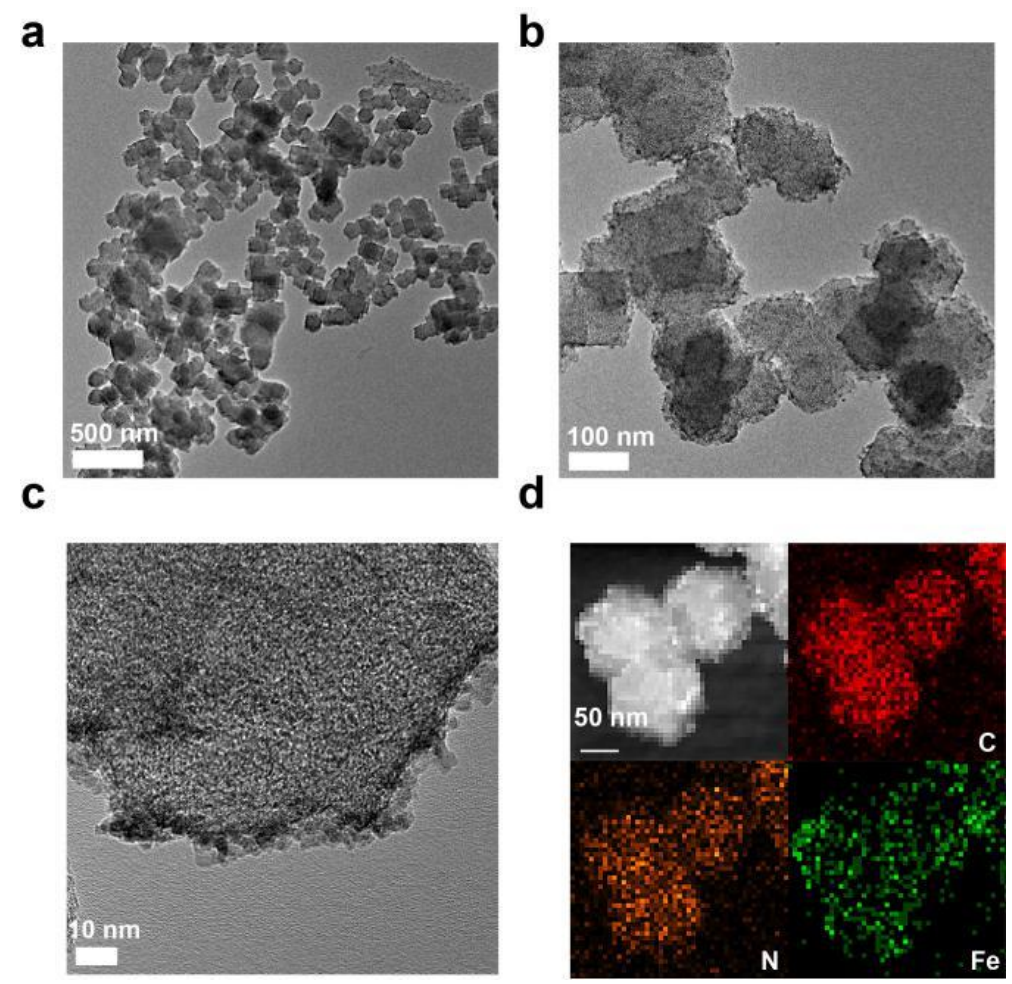

Figure 6. a, b) TEM images and c) HR-TEM image of PMCS-Fe under different magnifications. d) HAADF-STEM image of PMCS-Fe, corresponding element maps showing the distribution of C (red), $\mathrm{N}$ (orange) and Fe (green).

Obviously, Fe nanoparticles can be found on PMCS-Fe, which was different from Fe/PMCS. 

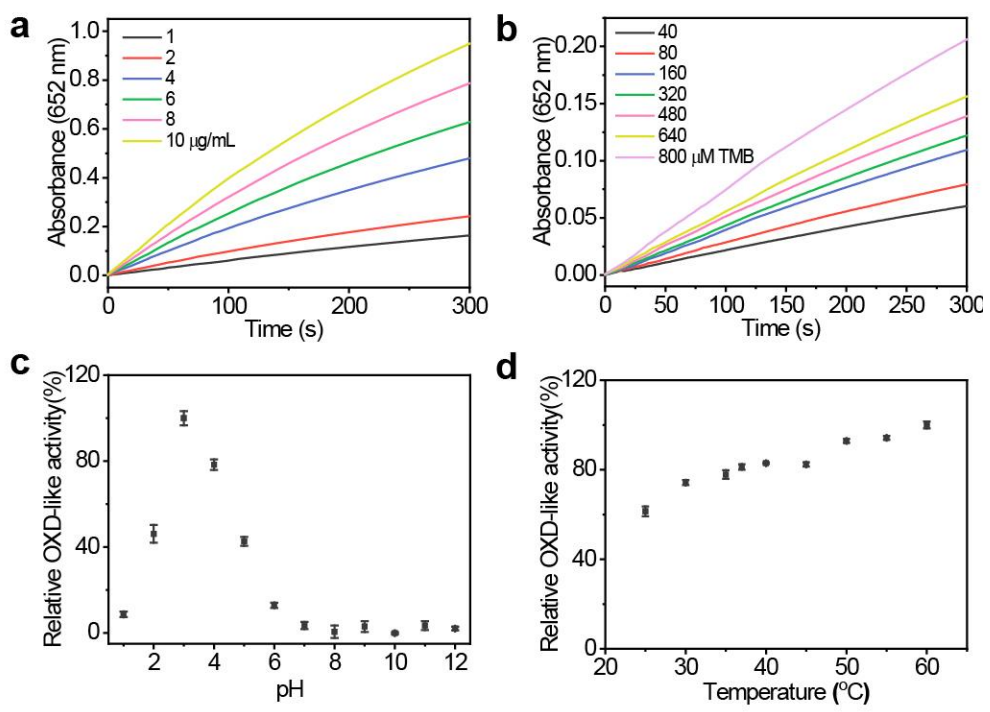

Figure 7. The OXD-like activity of Fe/PMCS is a) Fe/PMCS concentration, b) TMB concentration, c) $\mathrm{pH}$ and d) temperature dependent. Experiments were carried out using $2 \mu \mathrm{g} / \mathrm{mL}$ $\mathrm{Fe} / \mathrm{PMCS}$ in $500 \mu \mathrm{L}$ PB buffer $(20 \mathrm{mM}, \mathrm{pH} 3.0)$ at $37^{\circ} \mathrm{C}$ with $800 \mu \mathrm{M}$ TMB as substrate, unless otherwise stated. The maximum point in Supplementary Fig. 7c and 7d was set as 100\%. 
a

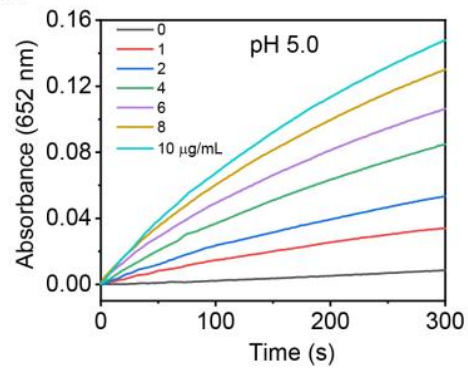

b

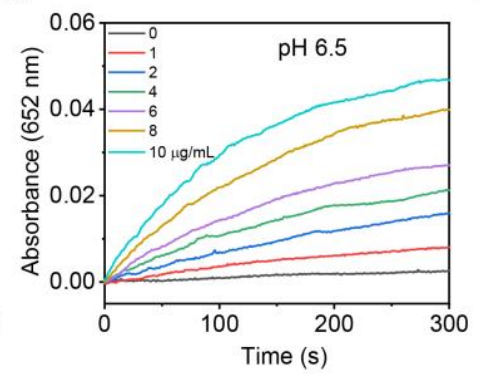

C

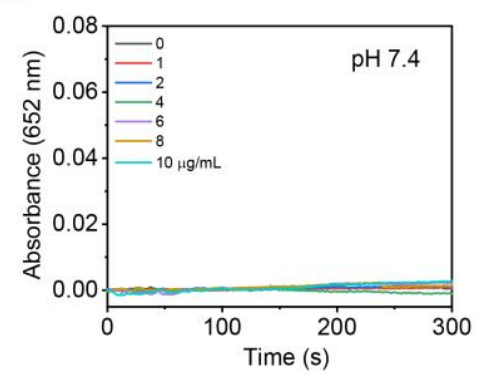

Figure 8. OXD-like activity of Fe/PMCS in slightly acidic environment of a) $\mathrm{pH} 5.0$ (mimicking the lysosome), b) pH 6.5 (mimicking the tumor microenvironment) and c) $\mathrm{pH} 7.4$ (mimicking the physiological environment). 

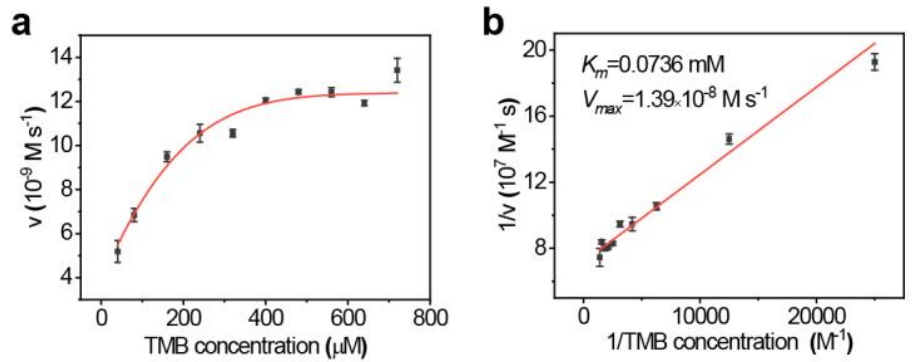

Figure 9. OXD-like activity of Fe/PMCS. a) Michaelis-Menten and b) corresponding Lineweaver-Burk plot with various concentrations of TMB $(40-720 \mu \mathrm{M})$ for Fe/PMCS. The data followed the Michaelis-Menten model and $K_{m}$ was determined as $0.0736 \mathrm{mM}$ for TMB, indicating a higher affinity for TMB. Besides, the $V_{\max }$ value was $1.39 \times 10^{-8} \mathrm{M} / \mathrm{s}$ for TMB. 

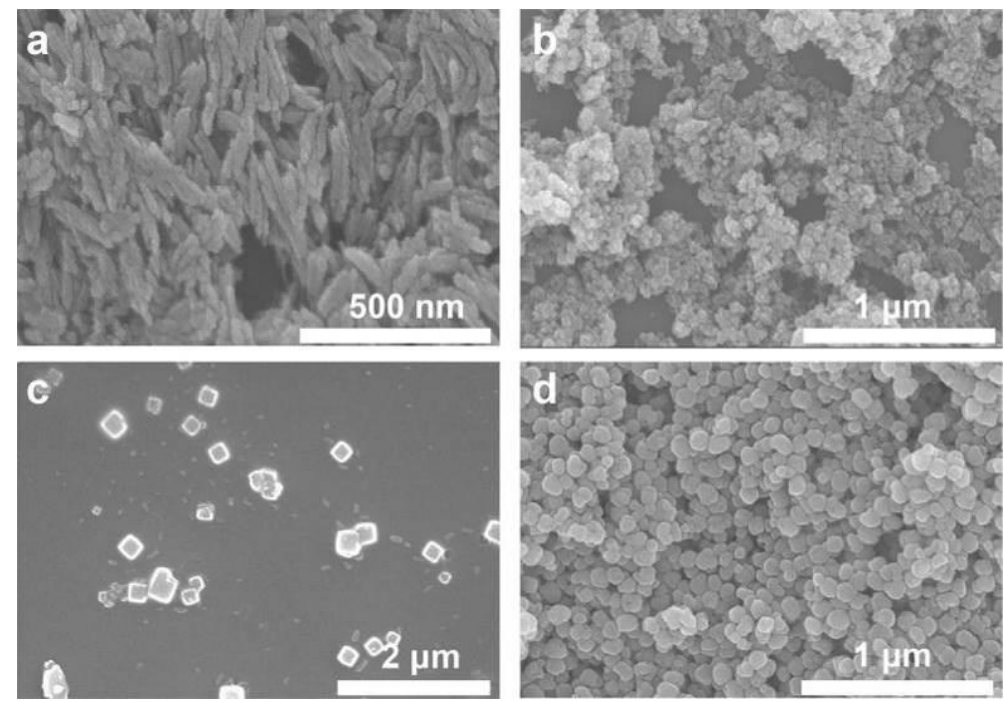

Figure 10. SEM images of a) $\alpha-\mathrm{Fe}_{2} \mathrm{O}_{3}$, b) $\gamma-\mathrm{Fe}_{2} \mathrm{O}_{3}$, c) $\mathrm{PB}$ and d) $\mathrm{Fe}_{3} \mathrm{O}_{4}$. 

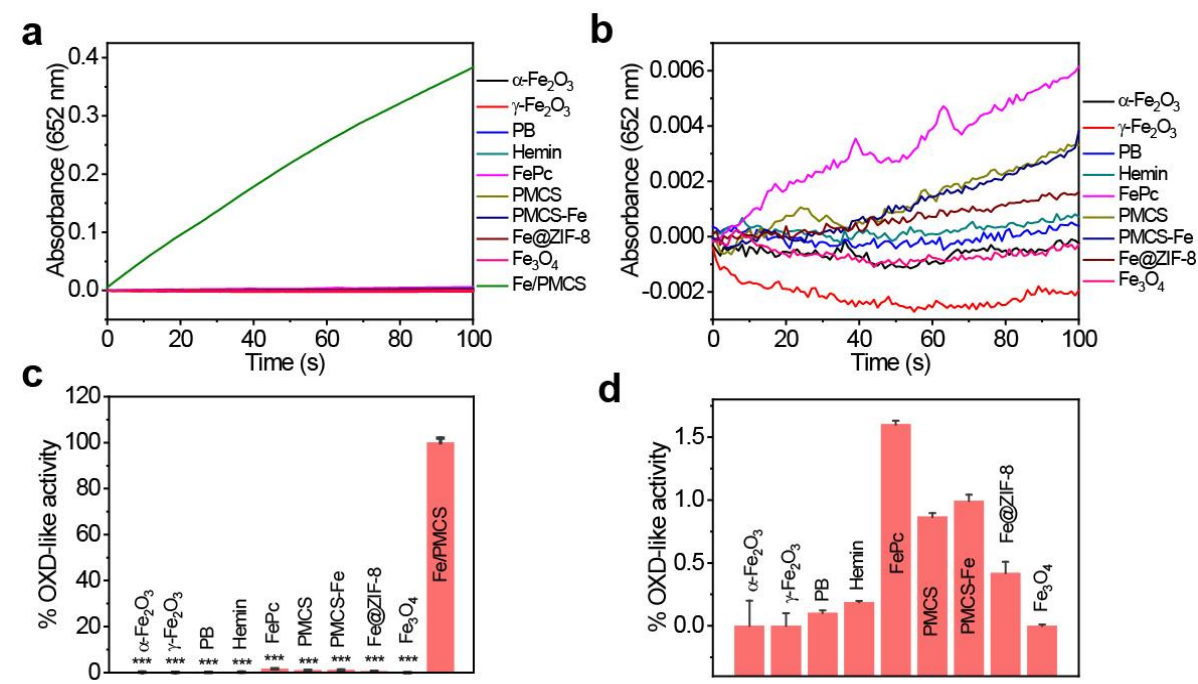

d

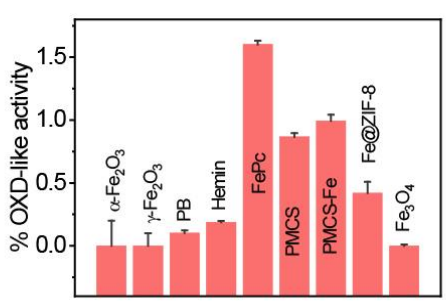

Figure 11. Comparison of the OXD-like activities of Fe/PMCS with other Fe-based nanozymes. a, b) The OXD-like activity of various nanozymes by monitoring the absorption of TMB at $652 \mathrm{~nm}$ and $\mathrm{c}, \mathrm{d}$ ) the comparison of their initial reaction rates. The maximum initial reaction rate was set as $100 \%$. The concentration of $\mathrm{Fe}$ was $3 \times 10^{-6} \mathrm{M}$, while the concentration of PMCS was 10 $\mu \mathrm{g} / \mathrm{mL} . * P<0.05, * * P<0.01$ and $* * * P<0.001$ versus the Fe/PMCS. 

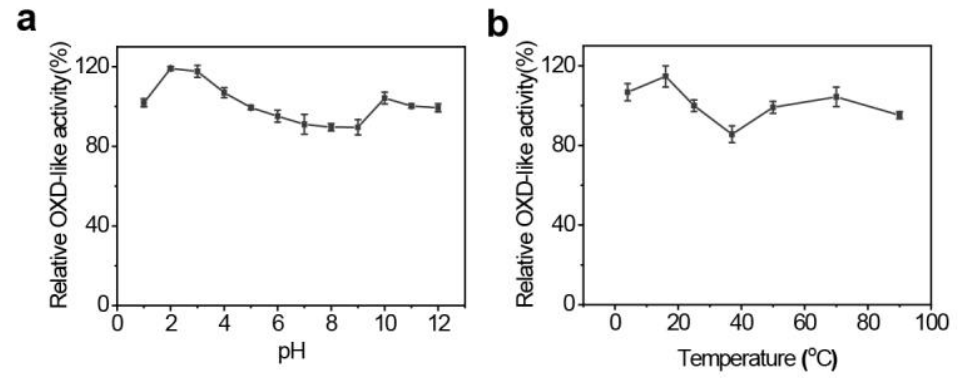

Figure 12. The stability of Fe/PMCS after incubated at different a) $\mathrm{pH}$ and b) temperature. 


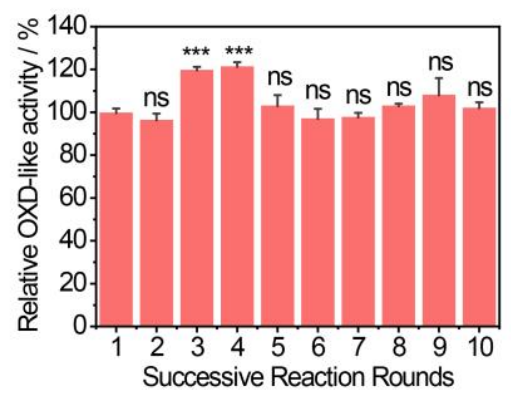

Figure 13. The relative OXD-like activity of the cycled Fe/PMCS. ns, no significance, ${ }^{*} P<0.05$, $* * P<0.01$ and $* * * P<0.001$ versus the original Fe/PMCS. Data were presented as mean \pm s.d. (n $=3)$. 
a

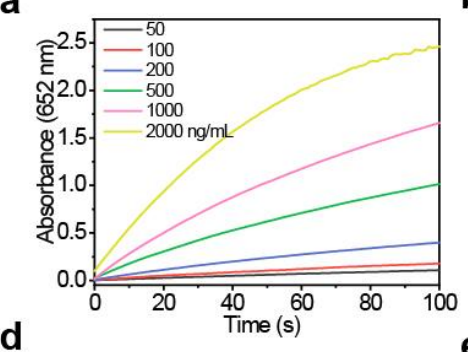

d

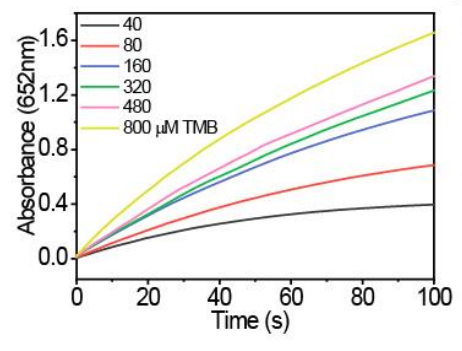

b
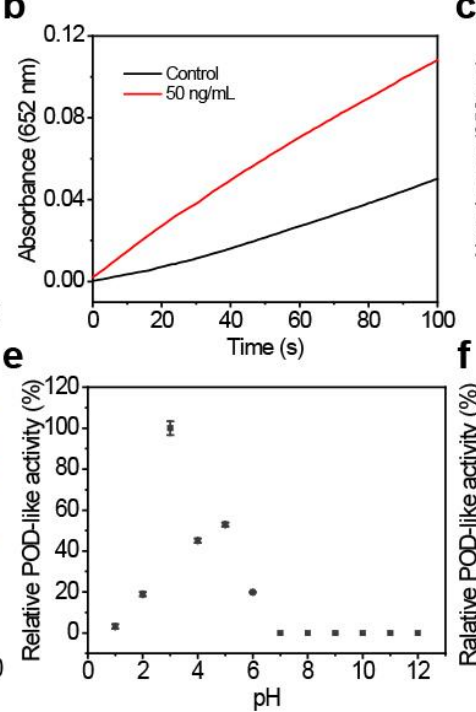

C
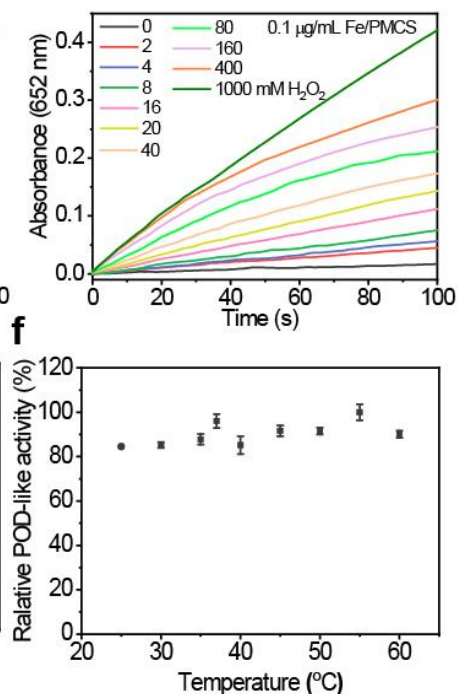

Figure 14. The POD-like activity of Fe/PMCS is a, b) Fe/PMCS concentration, c) $\mathrm{H}_{2} \mathrm{O}_{2}$ concentration, d) TMB concentration, e) $\mathrm{pH}$ and f) temperature dependent. Experiments were carried out using $1 \mu \mathrm{g} / \mathrm{mL} \mathrm{Fe} / \mathrm{PMCS}$ in $500 \mu \mathrm{L}$ PB buffer ( $20 \mathrm{mM}, \mathrm{pH} 3.0)$ at $37^{\circ} \mathrm{C}$ with $800 \mu \mathrm{M}$ TMB as substrate in the presence of $40 \mathrm{mM} \mathrm{H}_{2} \mathrm{O}_{2}$, unless otherwise stated. The maximum point in Supplementary Fig. 14e and $14 \mathrm{f}$ was set as $100 \%$. 

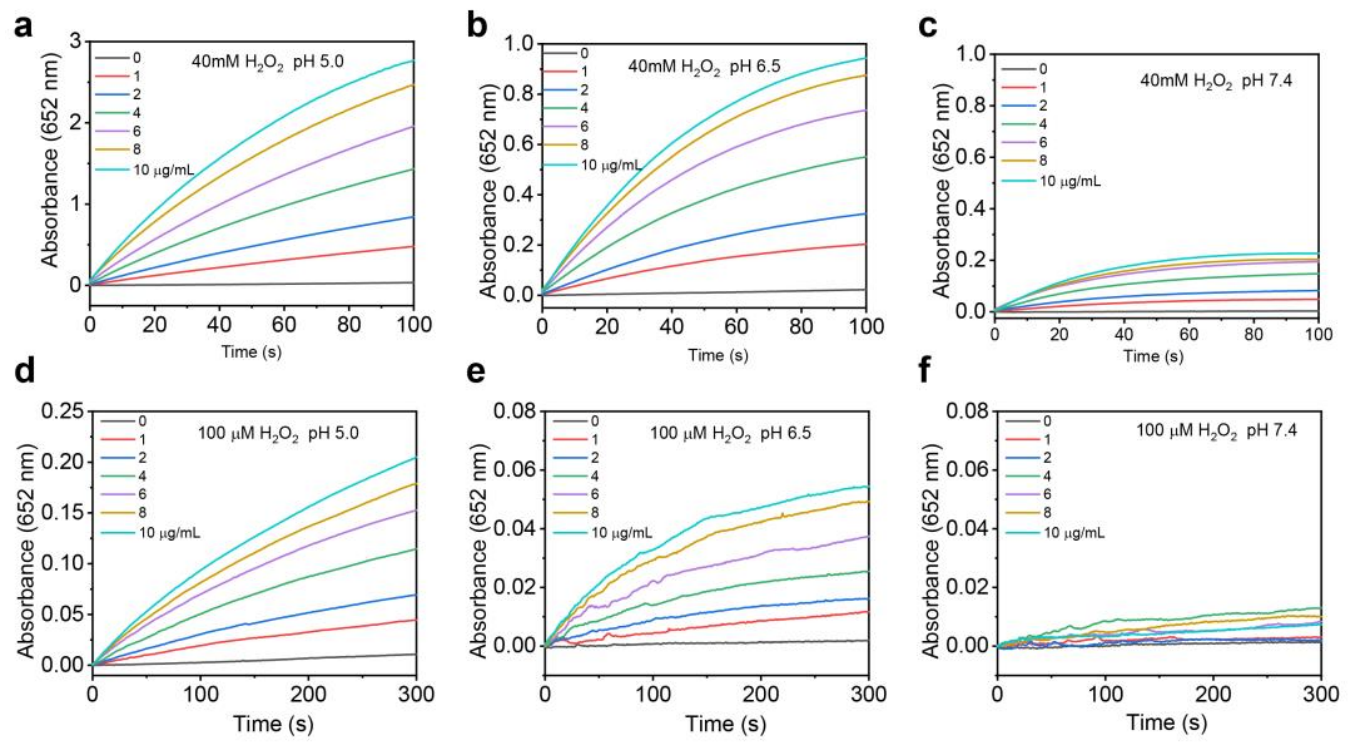

Figure 15. The POD-like activity of Fe/PMCS at pH 5.0, pH 6.5 and $\mathrm{pH} 7.4$ in the presence of 40 $\mathrm{mM}$ or $100 \mu \mathrm{M} \mathrm{H}_{2} \mathrm{O}_{2}$. 

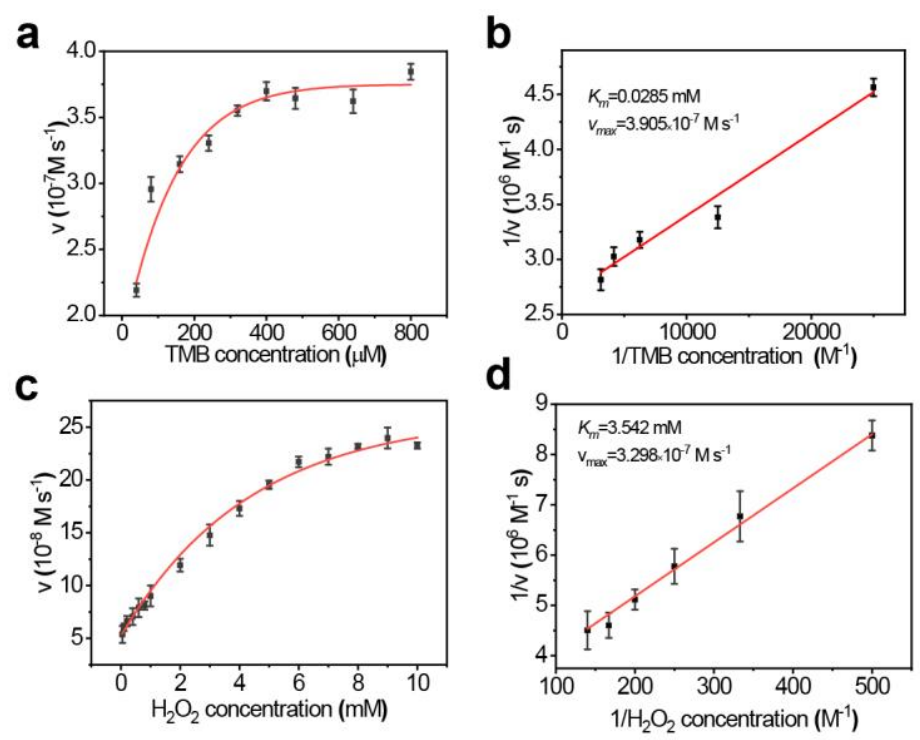

Figure 16. POD-like activity of Fe/PMCS. a) Michaelis-Menten and b) corresponding Lineweaver-Burk plot with various concentrations of TMB $(40-800 \mu \mathrm{M})$ for Fe/PMCS. c) Michaelis-Menten and d) corresponding Lineweaver-Burk plot with various concentrations of $\mathrm{H}_{2} \mathrm{O}_{2}(0.2-10 \mathrm{mM})$ for Fe/PMCS.

The data followed the Michaelis-Menten model and $K_{m}$ were determined as $0.0285 \mathrm{mM}$ for TMB and $3.54 \mathrm{mM}$ for $\mathrm{H}_{2} \mathrm{O}_{2}$, indicating a higher affinity for TMB and $\mathrm{H}_{2} \mathrm{O}_{2}$. Besides, the $V_{\max }$ value was $3.91 \times 10^{-7} \mathrm{M} / \mathrm{s}$ for TMB and $3.30 \times 10^{-7} \mathrm{M} / \mathrm{s}$ for $\mathrm{H}_{2} \mathrm{O}_{2}$. 

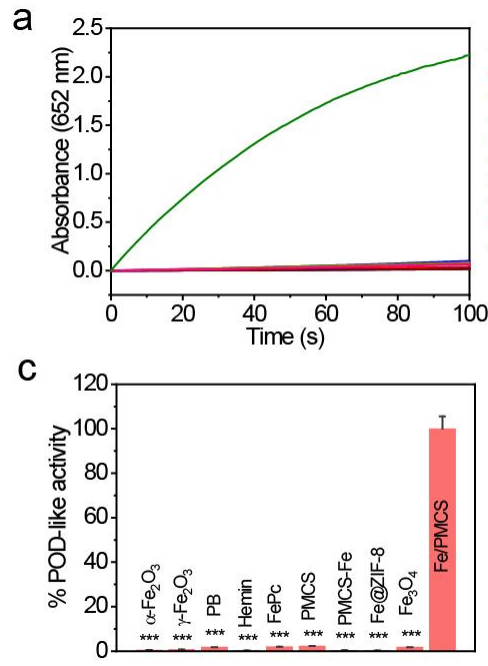

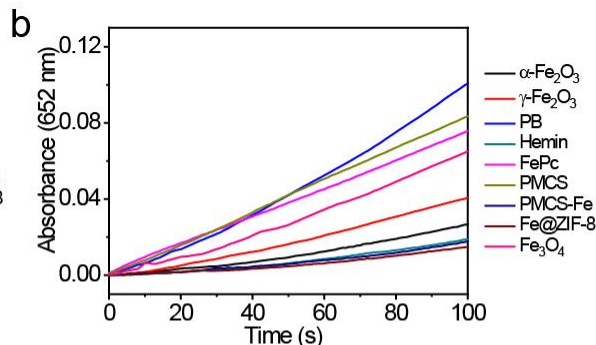

d

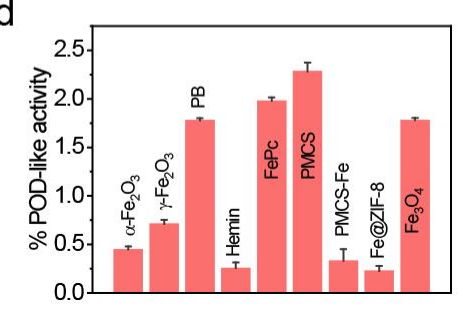

Figure 17. Comparison of the POD-like activities of Fe/PMCS with other Fe-based nanozymes. a, b) The POD-like activity of various nanozymes by monitoring the absorption of TMB at $652 \mathrm{~nm}$ and $\mathrm{c}, \mathrm{d}$ ) the comparison of their initial reaction rates. The maximum initial reaction rate was set as $100 \%$. The concentration of Fe was $6 \times 10^{-7} \mathrm{M}$, while the concentration of PMCS was $2 \mu \mathrm{g} / \mathrm{mL}$. $* P<0.05, * * P<0.01$ and $* * * P<0.001$ versus $\mathrm{Fe} / \mathrm{PMCS}$. 

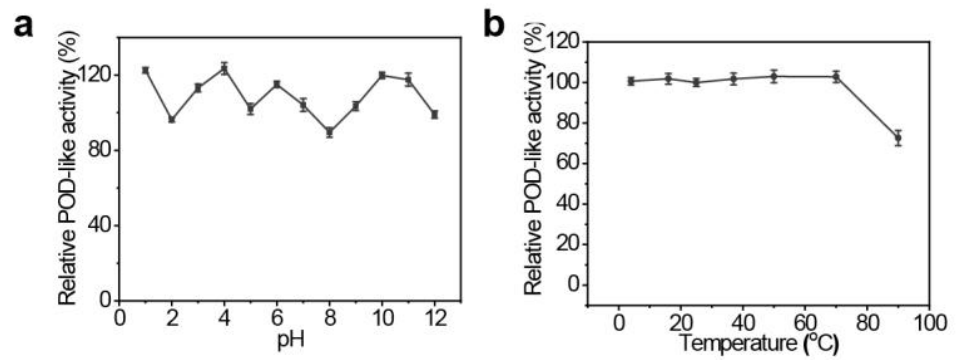

Figure 18. The stability of Fe/PMCS after incubated at different a) $\mathrm{pH}$ and b) temperature.

The drop of relative POD-like activity at higher temperature, i.e., $90{ }^{\circ} \mathrm{C}$ might be attributed the oxidation of Fe/PMCS, which has also observed in the other Fe-based POD-mimic. ${ }^{12}$ 


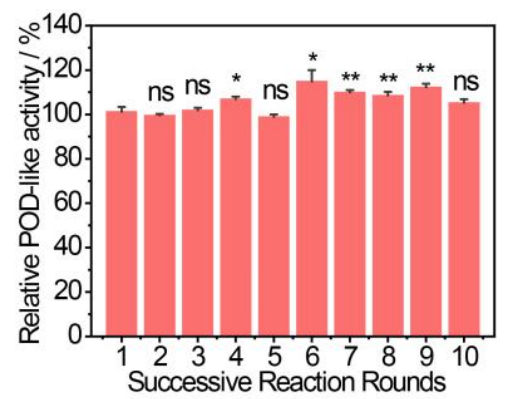

Figure 19. The relative POD-like activity of the cycled Fe/PMCS SAzymes. ns, no significance, ${ }^{*} P<0.05,{ }^{* *} P<0.01$ and ${ }^{* * *} P<0.001$ versus the original Fe/PMCS. Data were presented as mean \pm s.d. $(\mathrm{n}=3)$. 


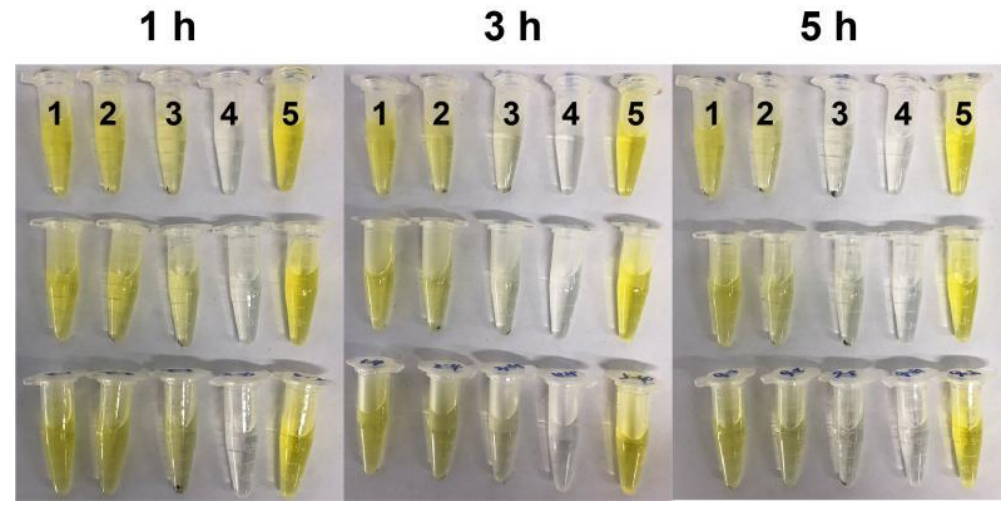

Figure 20. Photographs for the color change after GSH treatment at different time intervals, determined by the Ellman's assay. Tubes 1-3 in each group were the mixture of GSH with Fe/PMCS of 20, 40, $80 \mu \mathrm{g} / \mathrm{mL}$, respectively. Tubes 4 (positive control) was the mixture of GSH and $\mathrm{H}_{2} \mathrm{O}_{2}$. Tube 5 (negative control) was only GSH without adding $\mathrm{H}_{2} \mathrm{O}_{2}$ or Fe/PMCS. 


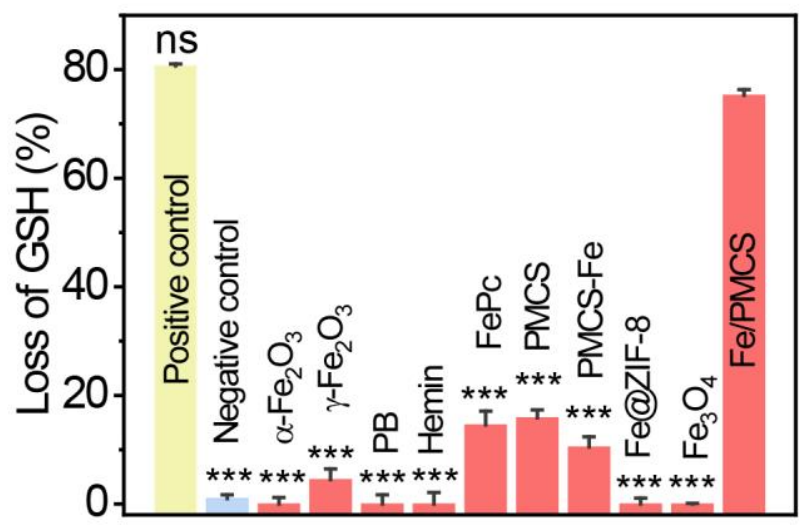

Figure 21. Comparison of the GSH-consuming abilities of Fe/PMCS with other Fe-based nanomaterials. The concentration of Fe was $2.4 \times 10^{-5} \mathrm{M}$, while the concentration of PMCS was $80 \mu \mathrm{g} / \mathrm{mL}$. ns, no significance, $* P<0.05, * * P<0.01$ and $* * * P<0.001$ versus $\mathrm{Fe} / \mathrm{PMCS}$. 

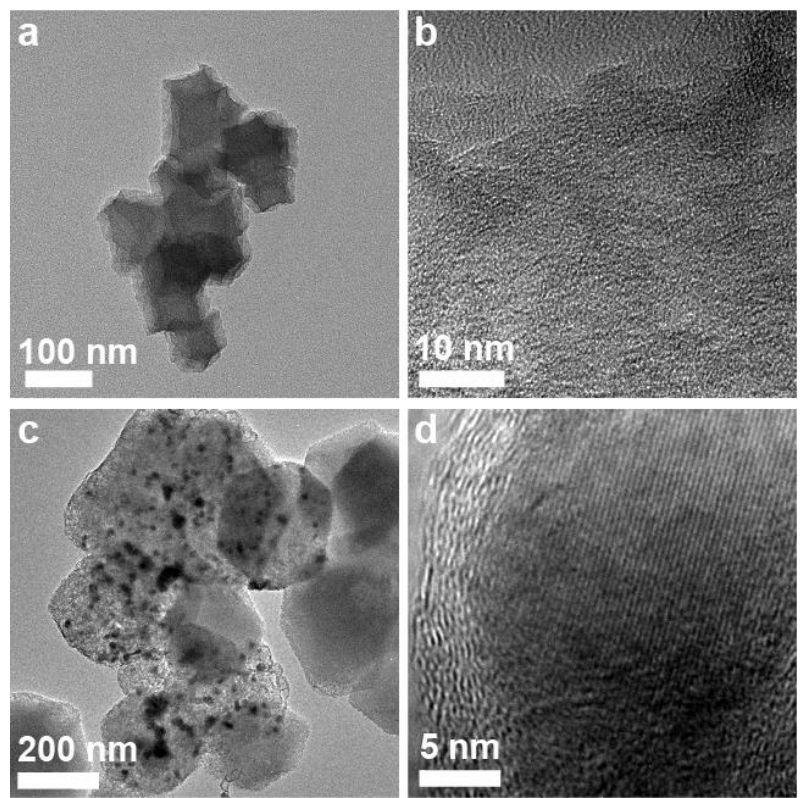

Figure 22. TEM of a, b) $\mathrm{Fe}_{0.1} / \mathrm{PMCS}$ and $\mathrm{c}$, d) $\mathrm{Fe}_{1.2} / \mathrm{PMCS}$ under different magnifications.

Notably, no nanoparticles could be found in $\mathrm{Fe}_{0.1} / \mathrm{PMCS}$, suggesting that $\mathrm{Fe}$ atoms might be confined in the carbon layers in an atomically dispersed form. Whereas, with the increase of Fe, there was distinct nanoparticles can be observed in $\mathrm{Fe}_{1.2} \mathrm{PMCS}$, indicating the presence of Fe-nanoparticles. Thus, the dose of Fe in Fe/PMCS structure could be manipulated by changing the contents of $\mathrm{Fe}(\mathrm{acac})_{3}$ in the synthesis of $\mathrm{Fe}(\mathrm{acac})_{3}$. In the sample of $\mathrm{Fe}_{x} / \mathrm{PMCS}$ (such as $\mathrm{Fe}_{0.1} / \mathrm{PMCS}, \mathrm{Fe}_{0.4} / \mathrm{PMCS}$ and $\left.\mathrm{Fe}_{1.2} / \mathrm{PMCS}\right)$, $\mathrm{x}$ represented the mole number of $\mathrm{Fe}(\mathrm{acac})_{3}$ in the synthesis of Fe@ZIF-8. 

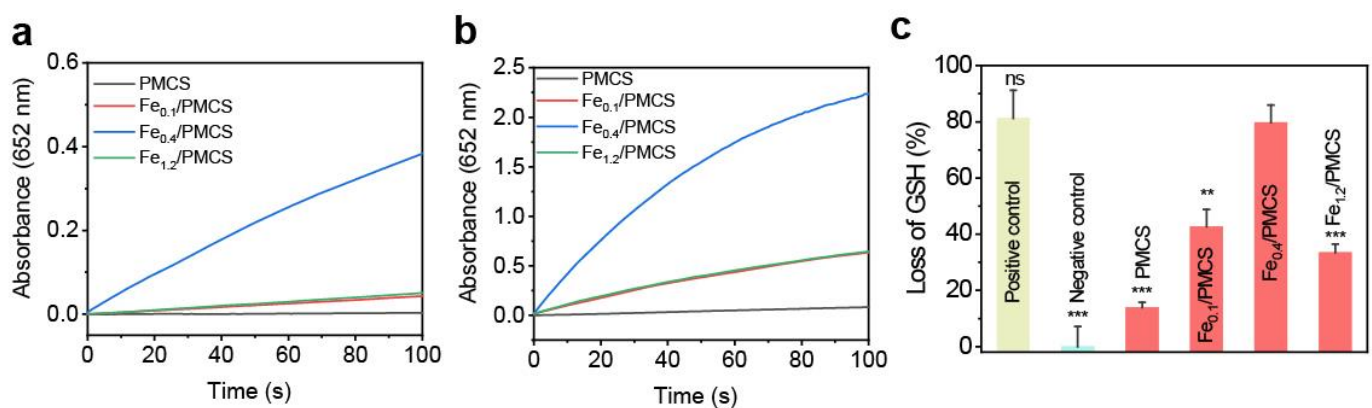

Figure 23. Comparison of the a) OXD-like, b) POD-like, and c) GSH-consuming of Fe/PMCS with different dose of Fe. ns, no significance, $* P<0.05$, $* * P<0.01$ and $* * * P<0.001$ versus $\mathrm{Fe}_{0.4} / \mathrm{PMCS}$.

Obviously, the dose of $\mathrm{Fe}$ in $\mathrm{Fe} / \mathrm{PMCS}$ structure could affect the catalytic activity of Fe/PMCS. Compared with $\mathrm{Fe}_{0.1} / \mathrm{PMCS}$ and $\mathrm{Fe}_{1.2} / \mathrm{PMCS}$, the activities of $\mathrm{Fe}_{0.4} / \mathrm{PMCS}$ significantly increased, indicating that the promotion of activity was attributed to maintaining the metal center Fe as atomically dispersed sites.

Thus, with the increase of Fe content in Fe/PMCS, the structure of Fe/PMCS will be changed from atomically dispersed form to nanoparticles. Meanwhile, the catalytic activity of Fe/PMCS could be promoted by increasing the metal center $\mathrm{Fe}$ as atomically dispersed sites. 
a

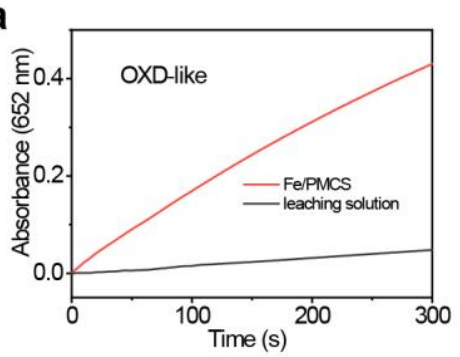

b

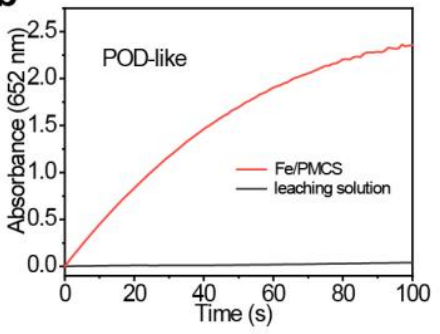

Figure 24. a) OXD-like and b) POD-like activities of Fe/PMCS and leaching solution. The concentration of Fe/PMCS was $10 \mu \mathrm{g} / \mathrm{mL}$ for OXD measurement, while $2 \mu \mathrm{g} / \mathrm{mL}$ for POD measurement. The leaching solution was derived from high concentrations of Fe/PMCS, in which the concentration was 100 times more than that of compared Fe/PMCS.

The leaching solution was inactive, while Fe/PMCS exhibited outstanding OXD-like and POD-like performance, demonstrating that the catalytic reaction was attributed to intact Fe/PMCS. Moreover, the Fe content in the supernatant was two orders of magnitude lower than that required for the Fenton reaction, ${ }^{1}$ further confirming that catalytic ability of Fe/PMCS was not derived from the leaching of Fe ions, but proceeded on the surface of Fe/PMCS. 


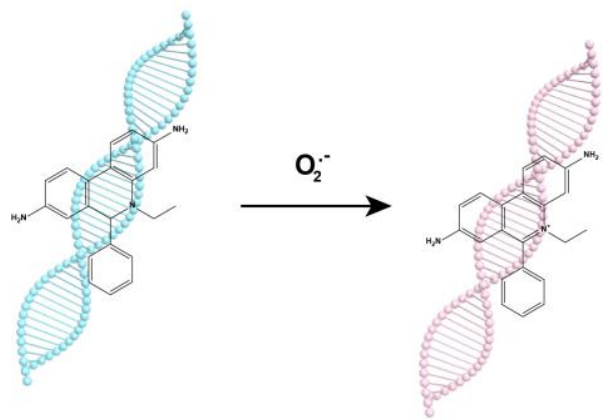

Figure 25. Illustration of detection of $\mathrm{O}_{2}{ }^{--}$by hydroethidine in the presence of DNA. 

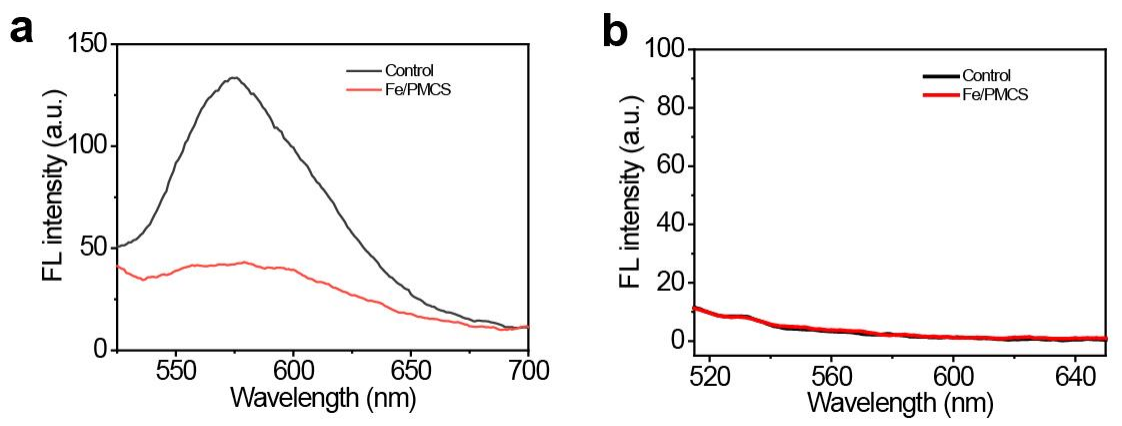

Figure 26. a) $\mathrm{O}_{2}{ }^{--}$and b) ${ }^{1} \mathrm{O}_{2}$ generation ability of Fe/PMCS.

Neither of them exhibited fluorescence enhancements when they incubated with Fe/PMCS, demonstrating that their incapability to produce $\mathrm{O}_{2}{ }^{-}$and ${ }^{1} \mathrm{O}_{2}$ 
a

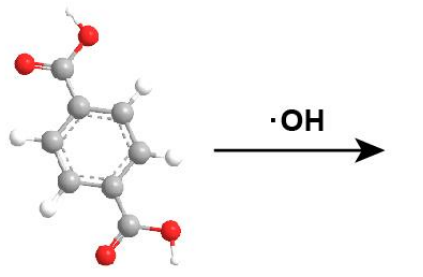

b

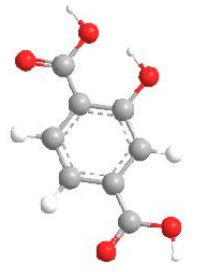

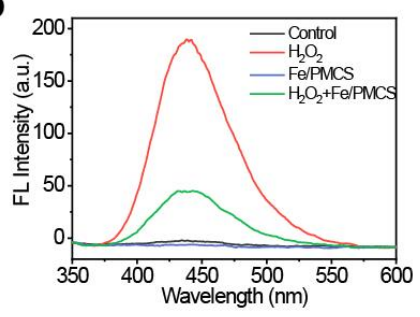

Figure 27. a) Illustration of the reaction between $\bullet \mathrm{OH}$ and TA. b) $\bullet \mathrm{OH}$ generation ability of Fe/PMCS.

Unfortunately, there was scarcely any increase in fluorescence intensity of 2-hydroxyterephtthalic acid (HA) to be observed, suggesting the invalid of $\bullet \mathrm{OH}$ production。 


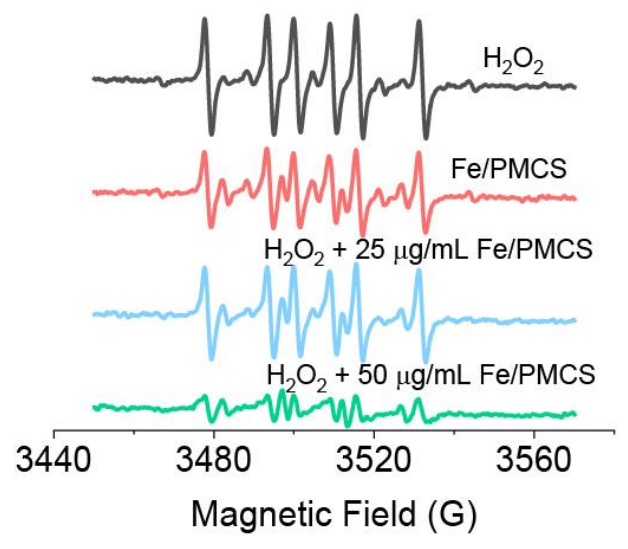

Figure 28. ESR spectra of $\mathrm{Fe} / \mathrm{PMCS}(0,25,50 \mu \mathrm{g} / \mathrm{mL})$ showing the invalid generation of $\bullet \mathrm{OH}$

No apparent $\mathrm{BMPO} / \bullet \mathrm{OH}$ signal was observed in the $\mathrm{H}_{2} \mathrm{O}_{2}$ solution regardless of the presence of Fe/PMCS, consistent with the negligible fluorescence enhancement of HA. Both of the fluorescence and ESR results demonstrated that the catalytic mechanism of POD-like activities was not to generate the active intermediate of $\bullet \mathrm{OH}$, which was accordant with previous studies. ${ }^{11,12-14}$ 
a

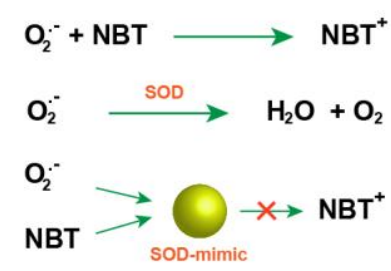

b

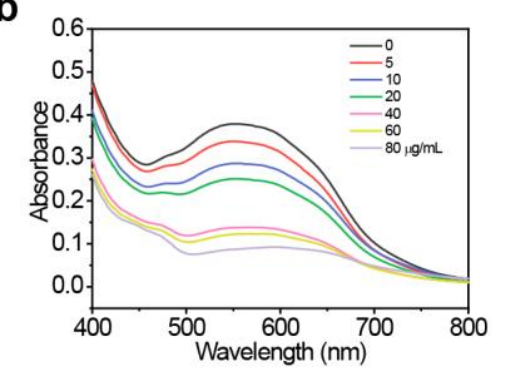

Figure 29. SOD-mimic ability of Fe/PMCS. a) The schematic illustration for the SOD-like activity. b) $\mathrm{O}_{2}{ }^{\circ}$-scavenging ability of Fe/PMCS. Percent inhibition of NBT oxidation with different concentrations of Fe/PMCS in PB buffer (10 mM, pH 7.4). Fe/PMCS: 0-80 $\mu \mathrm{g} / \mathrm{mL}$, riboflavin: $20 \mu \mathrm{M}$, methionine: $13 \mathrm{mM}$, NBT: $75 \mu \mathrm{M}$. 


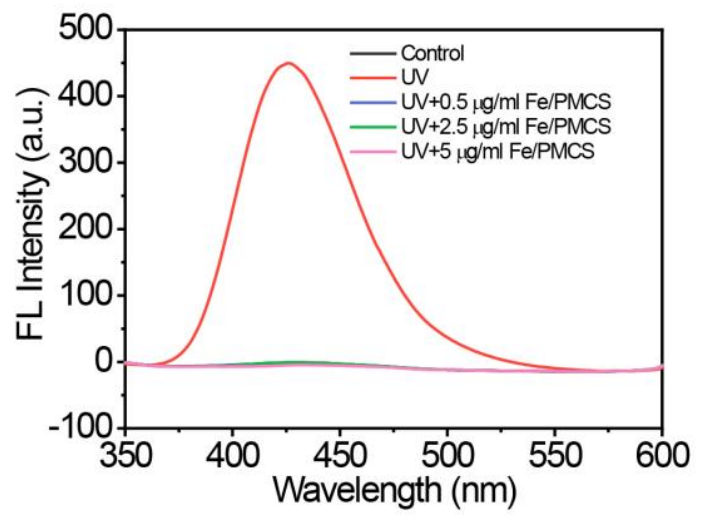

Figure 30. $\cdot \mathrm{OH}$-scavenging ability of Fe/PMCS.

Fluorescence spectra showed the scavenging efficiency of $\bullet \mathrm{OH}$ in the presence of different concentrations of Fe/PMCS in PB buffer (10 mM, pH 7.4). Fe/PMCS: 0.5-5 $\mu \mathrm{g} / \mathrm{mL}, \mathrm{H}_{2} \mathrm{O}_{2}: 1 \mathrm{mM}$, TA: $0.5 \mathrm{mM}$. 


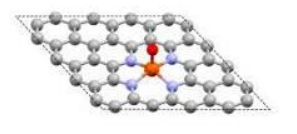

int11

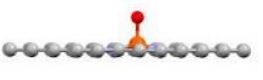

int11-F

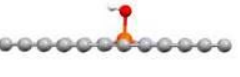

int12-F

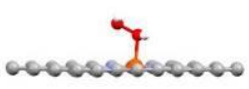

ts11-F

ts11

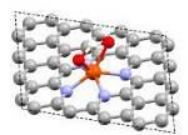

ts12

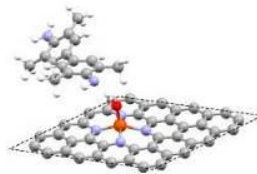

ts13

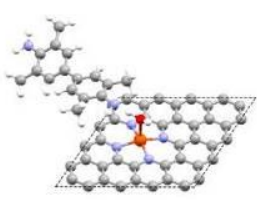

ts14

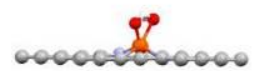

ts12-F

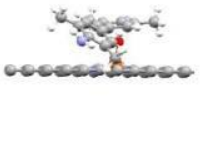

ts13-F

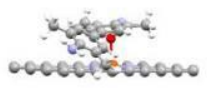

ts14-F
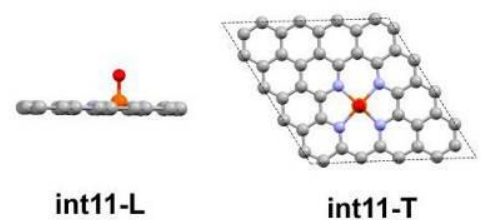

int11-T
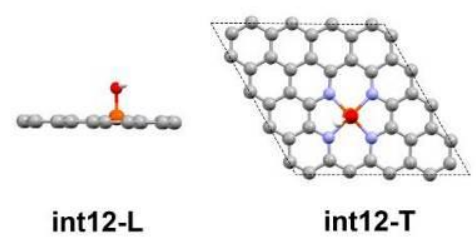

int12-T

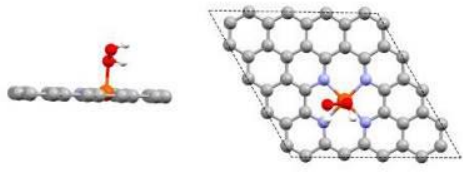

ts11-L

ts11-T

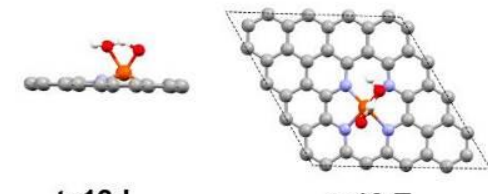

ts12-L

ts12-T

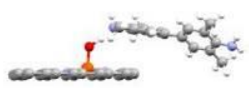

ts13-L

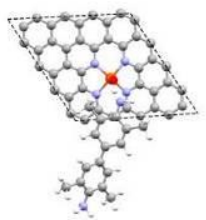

ts13-T

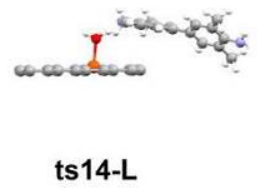

Figure 31. Proposed optimized structures of intermediates for OXD-like activity. The orange, blue, gray, red and white balls represent the Fe, N, C, O and $\mathrm{H}$ atoms, respectively. F: front view. L: left view. T: top view. 


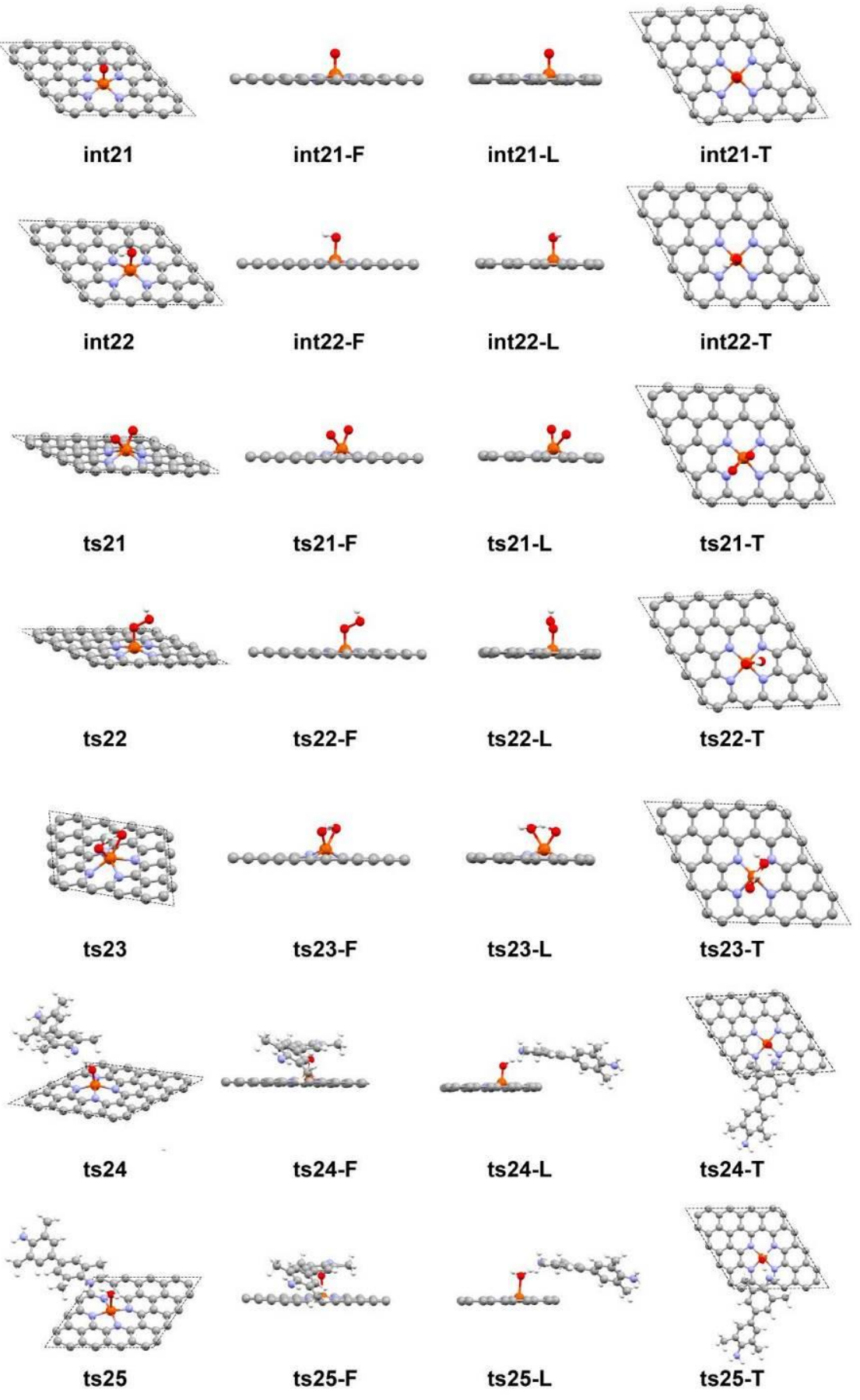

Figure 32. Proposed optimized structures of intermediates for POD-like activity. 


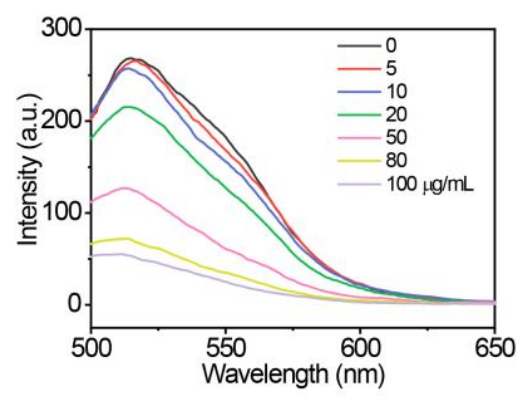

Figure 33. Fluorescence spectra of FAM-mDNA responding to Fe/PMCS at increased concentrations. 


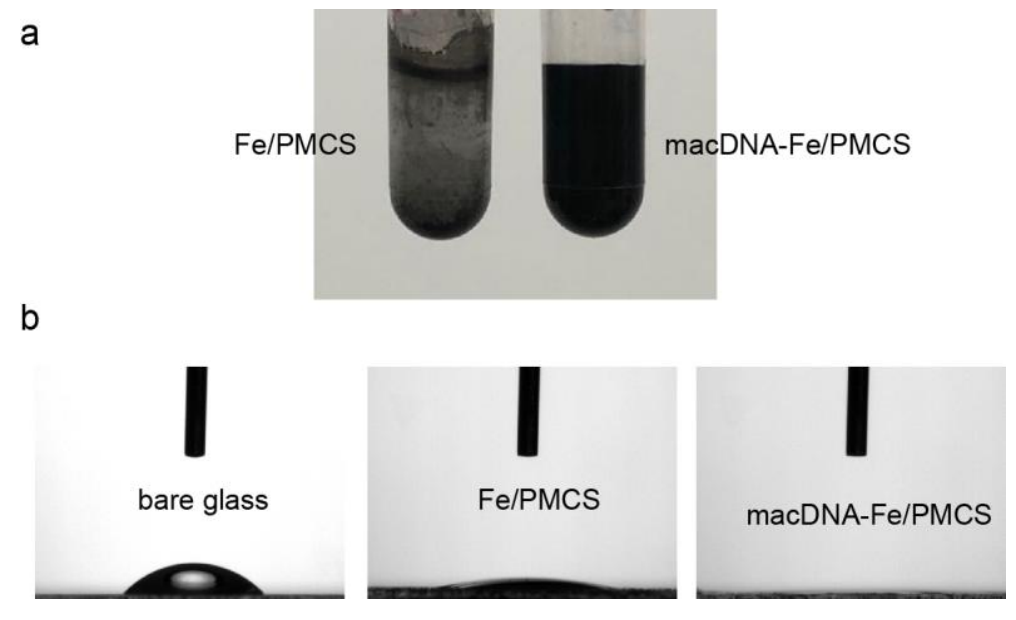

Figure 34. a) Photograph of Fe/PMCS and macDNA-Fe/PMCS with a concentration of 500 $\mu \mathrm{g} / \mathrm{mL}$ in PBS for $24 \mathrm{~h}$. b) Water contact angles on bare glass, Fe/PMCS and macDNA-Fe/PMCS.

The modification DNA increased the hydrophilicity of Fe/PMCS, suggested by the good solubility in PBS even after $24 \mathrm{~h}$ of incubation and the smaller water angles. 

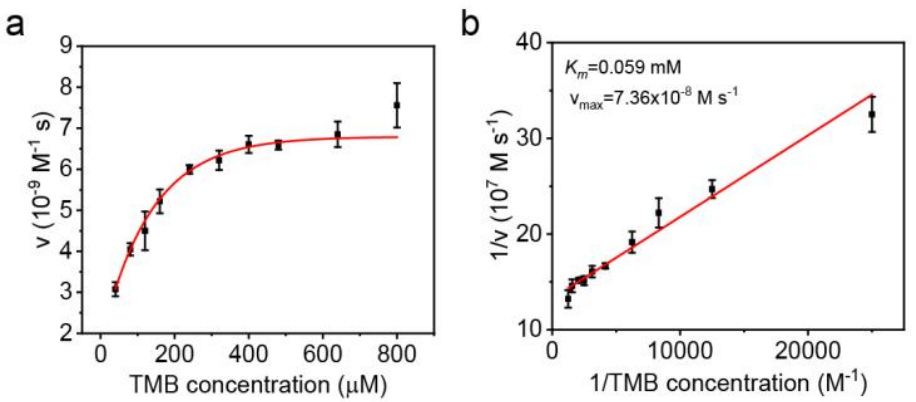

Figure 35. OXD-like activity of macDNA-Fe/PMCS. a) Michaelis-Menten and b) corresponding Lineweaver-Burk plot with various concentrations of TMB $(40-800 \mu \mathrm{M})$ for $1 \mu \mathrm{g} / \mathrm{mL}$ macDNA-Fe/PMCS. 

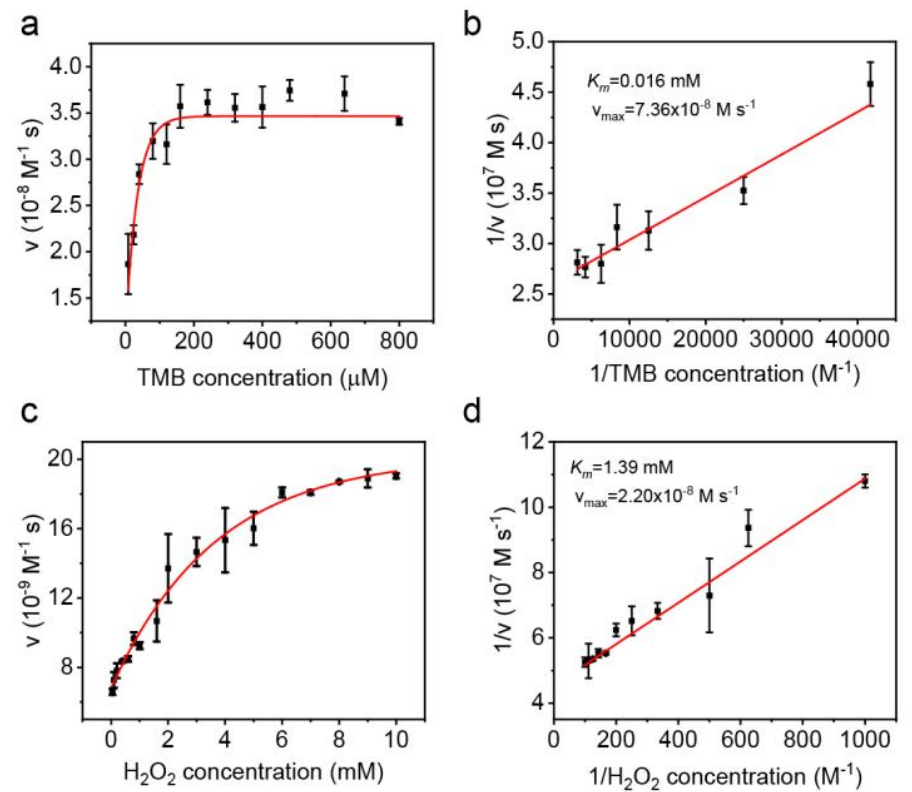

Figure 36. POD-like activity of macDNA-Fe/PMCS. a) Michaelis-Menten and b) corresponding Lineweaver-Burk plot with various concentrations of TMB $(40-800 \mu \mathrm{M})$ for $1 \mu \mathrm{g} / \mathrm{mL}$ macDNA-Fe/PMCS. c) Michaelis-Menten and d) corresponding Lineweaver-Burk plot with various concentrations of $\mathrm{H}_{2} \mathrm{O}_{2}(0.05-10 \mathrm{mM})$ for $1 \mu \mathrm{g} / \mathrm{mL}$ macDNA-Fe/PMCS. 
a

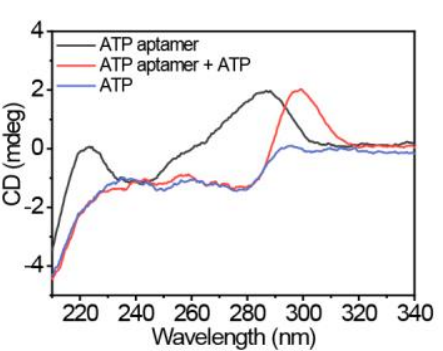

b

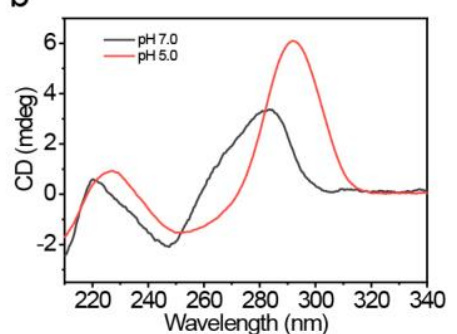

Figure 37. a) CD spectra of ATP aptamer, ATP, the mixture of ATP aptamer and ATP at pH 7.0. b) CD spectra of C-rich DNA at $\mathrm{pH} 5.0$ and $\mathrm{pH}$ 7.0. 


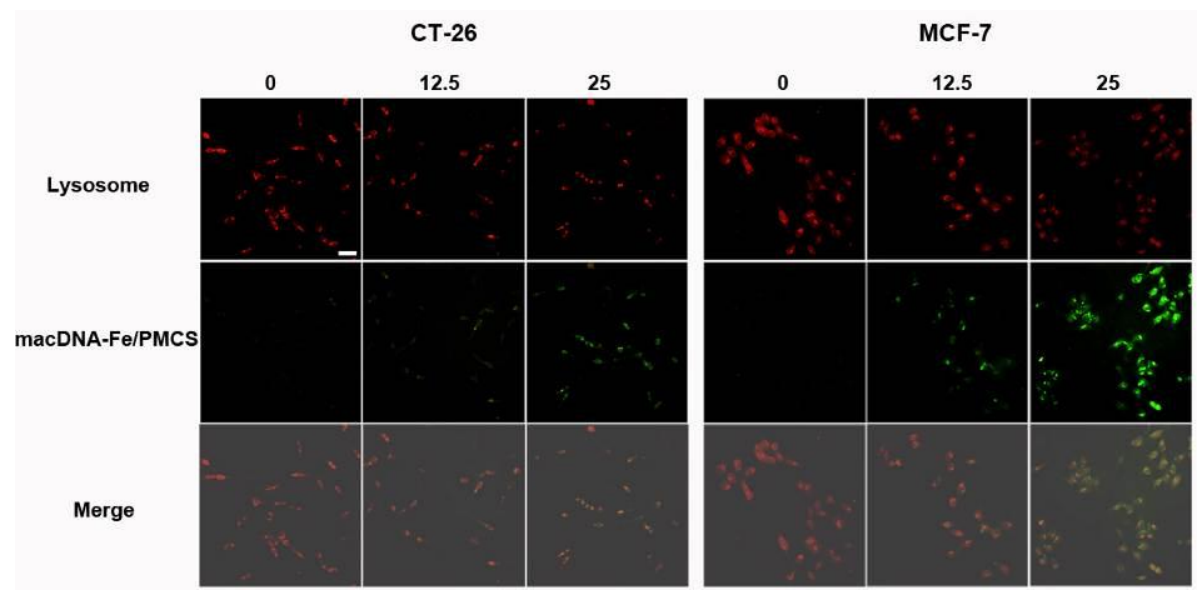

Figure 38. Fluorescence microscope images of CT-26 and MCF-7 cancer cells incubated with different concentration of FAM labeled macDNA-Fe/PMCS for $4 \mathrm{~h}$ at $37{ }^{\circ} \mathrm{C}$. The series can be classified as the lysosomes of cells (dyed red by LysoTracker for visualization), macDNA-Fe/PMCS (green) and a merge of the abovementioned two channels. Scale bar is $50 \mu \mathrm{m}$. 

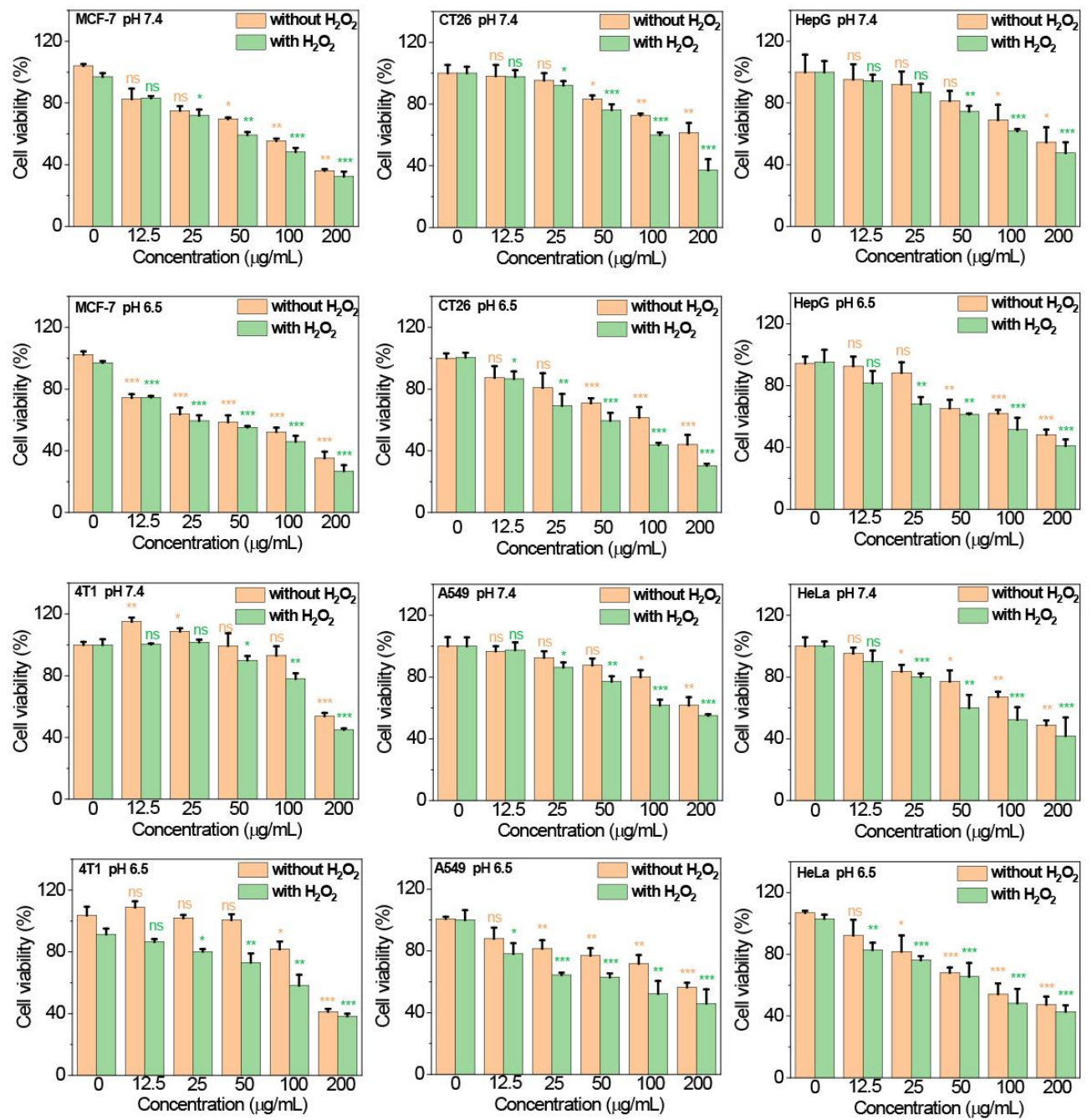

Figure 39. The cell viability of different cancer cells treated with macDNA-Fe/PMCS with or without $\mathrm{H}_{2} \mathrm{O}_{2}$ at $\mathrm{pH} 7.4$ or $\mathrm{pH} 6.5$. ns, no significance, ${ }^{*} P<0.05$, ${ }^{* *} P<0.01$ and ${ }^{* * *} P<0.001$ versus macDNA-Fe/PMCS untreated cells. 

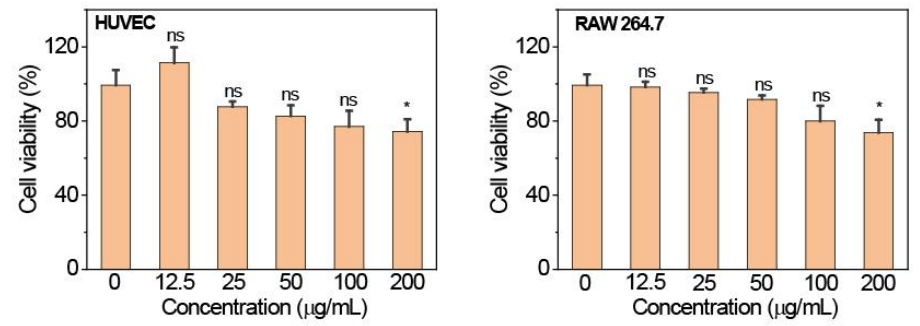

Figure 40. The cell viability of different normal cells treated with macDNA-Fe/PMCS at pH 7.4. ns, no significance, $* P<0.05, * * P<0.01$ and $* * * P<0.001$ versus untreated cells. 


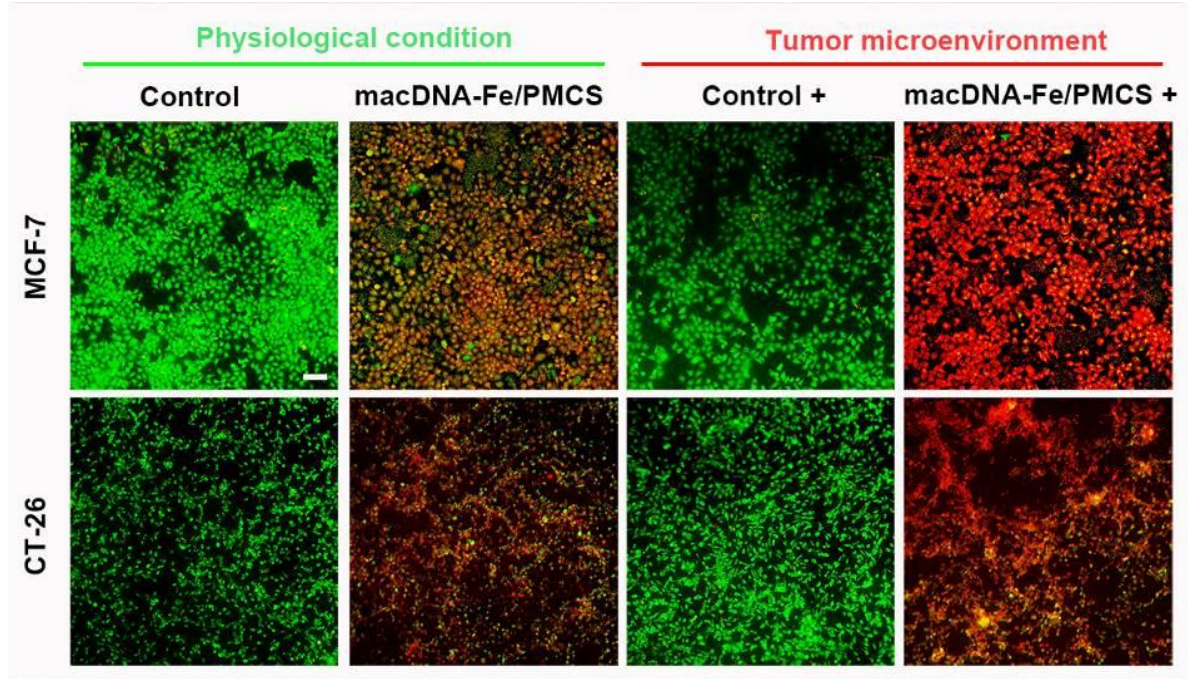

Figure 41. LIVE/DEAD staining of a) MCF-7 and b) CT-26 cells incubated with macDNA-Fe/PMCS in both normal and tumor microenvironment. Scale bars are $100 \mu \mathrm{m}$. 

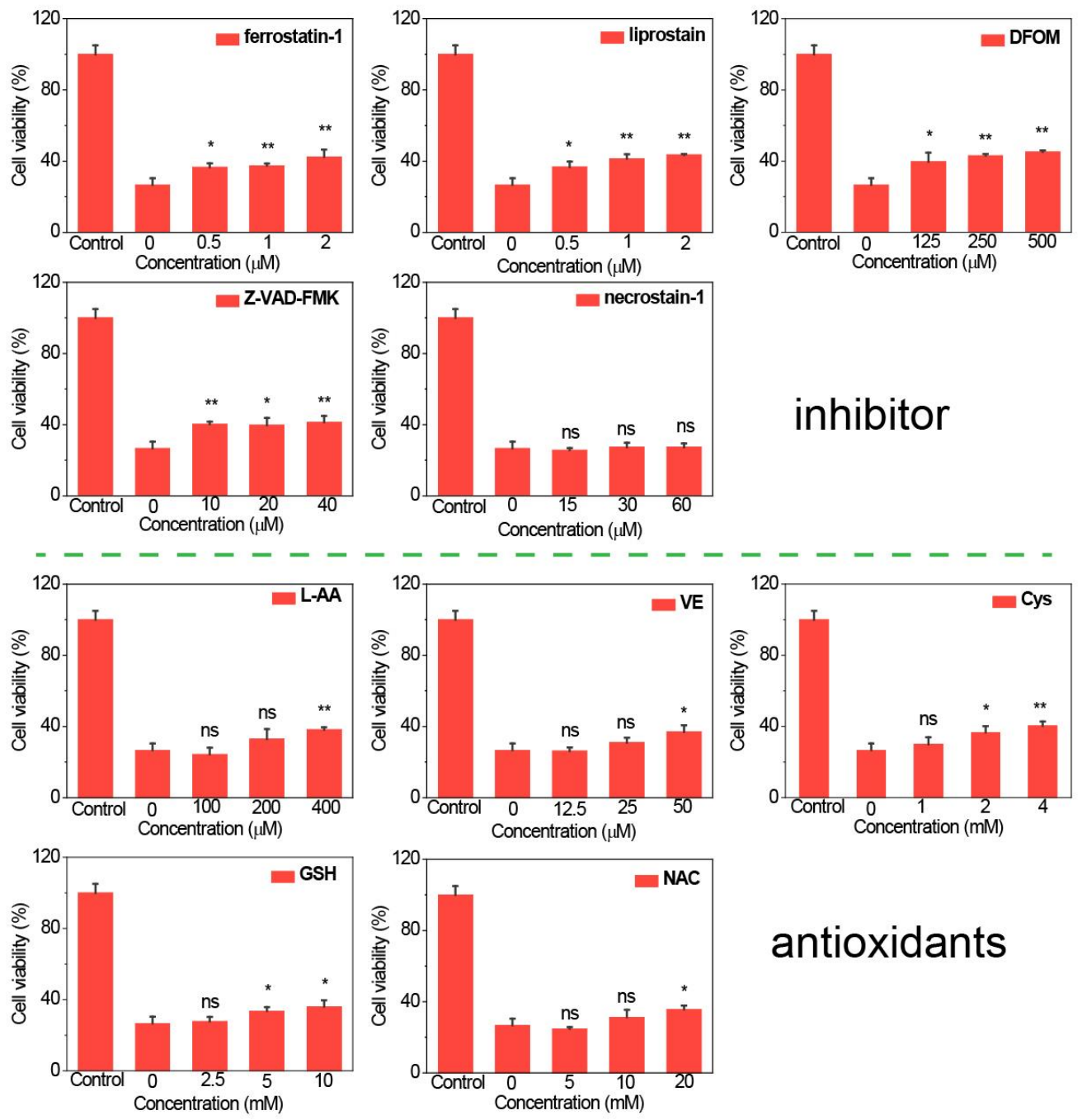

\section{inhibitor}

\section{antioxidants}

Figure 42. The cell viability of MCF-7 cells treated with macDNA-Fe/PMCS and inhibitor of ferrostatin-1, liprostain, Z-VAD-FMK, necrostain, or iron chelating agent of DFOM, or antioxidants of L-AA, VE, Cys, GSH and NAC. ns, no significance, $* P<0.05, * * P<0.01$ and $* * * P<0.001$ versus untreated groups. 

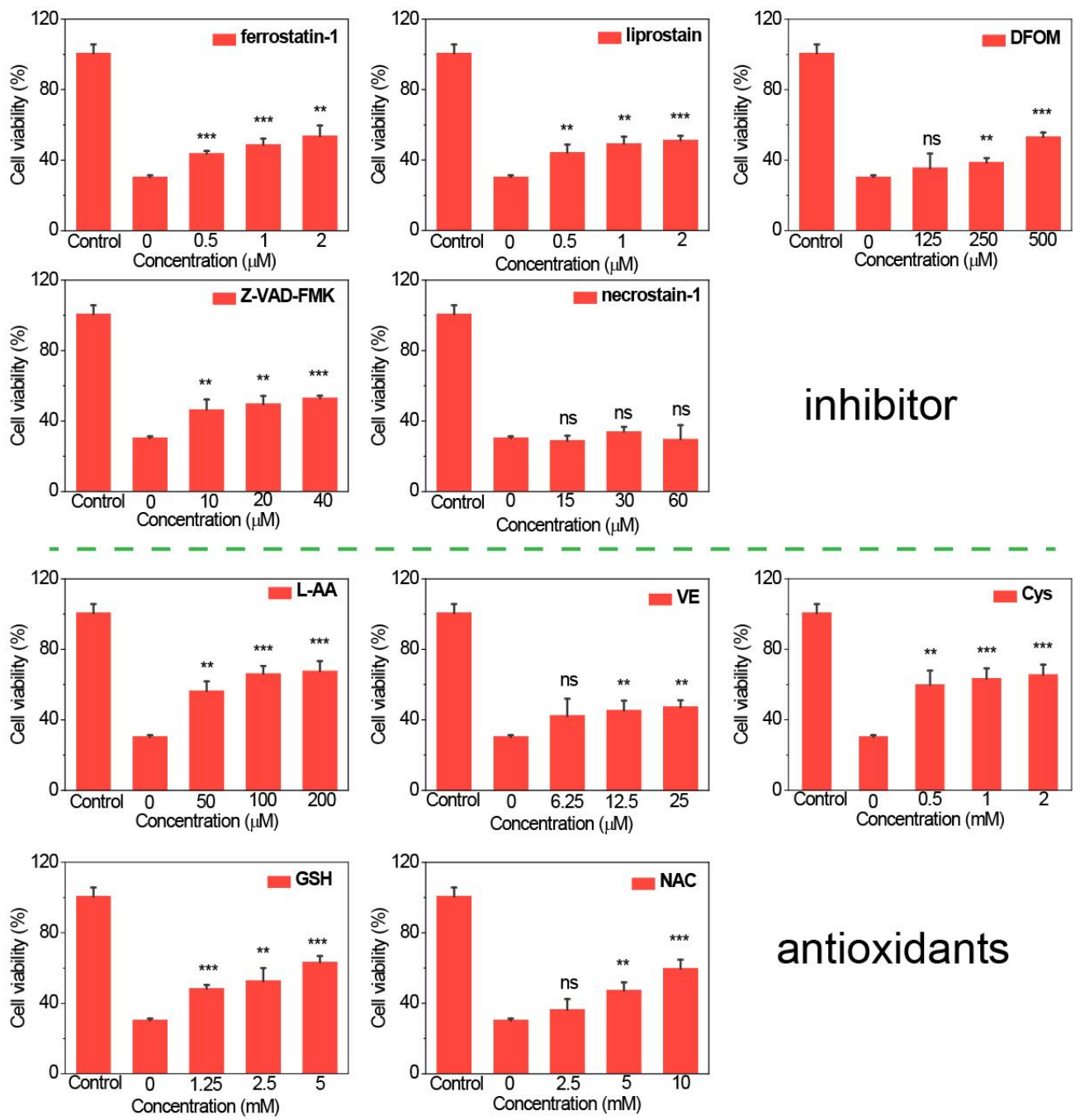

\section{antioxidants}

Figure 43. The cell viability of CT-26 cells treated with macDNA-Fe/PMCS and inhibitor of ferrostatin-1, liprostain, Z-VAD-FMK, necrostain, or iron chelating agent of DFOM, or antioxidants of L-AA, VE, Cys, GSH and NAC. ns, no significance, $* P<0.05, * * P<0.01$ and $* * * P<0.001$ versus untreated groups. 


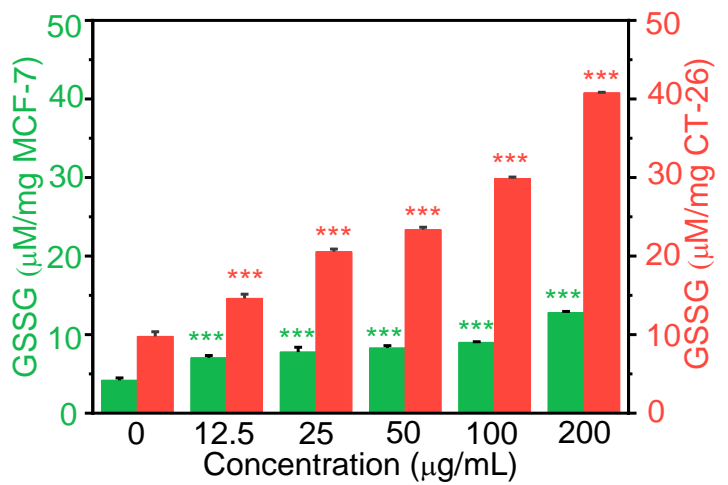

Figure 44. The GSSG contents measured after treatment with macDNA-Fe/PMCS as the concentration increase. $* P<0.05, * * P<0.01$ and $* * * P<0.001$ versus untreated groups. 


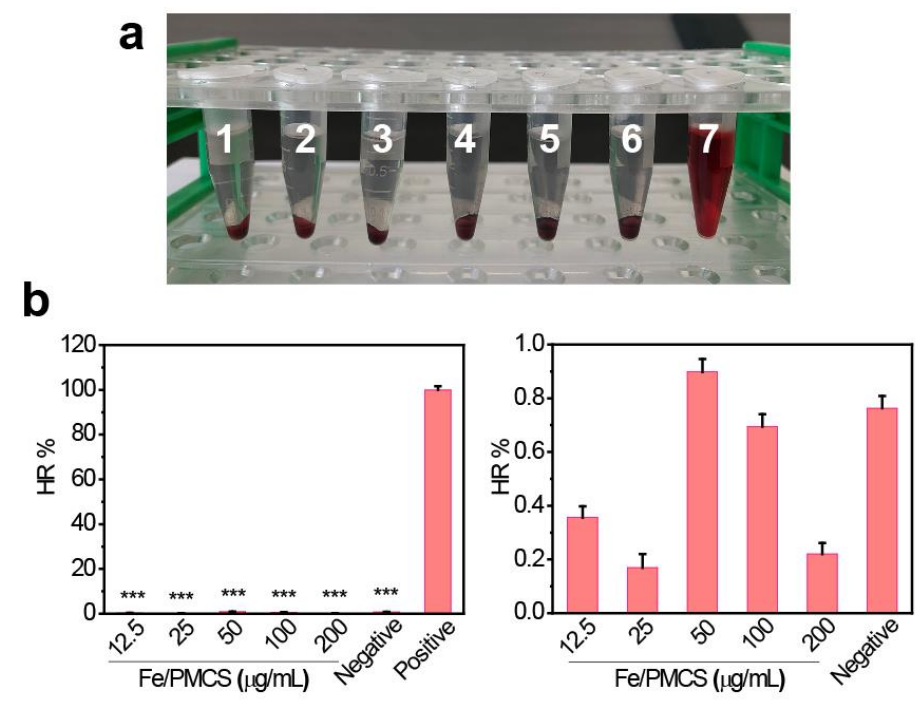

Figure 45. a) Digital photograph of hemolysis test and b, c) corresponding hemolysis rate (HR\%) of macDNA-Fe/PMCS. Tubes 1-5 was the mixture of erythrocyte suspension with macDNA-Fe/PMCS of 12.5, 25, 50, 100, $200 \mu \mathrm{g} / \mathrm{mL}$. Tube 6 (negative control) was placed with erythrocyte suspension diluted with $0.9 \%$ saline, tube 7 (positive control) was placed with erythrocyte suspension diluted with ultrapure water. $* P<0.05$, $* * P<0.01$ and $* * * P<0.001$ versus $\mathrm{H}_{2} \mathrm{O}$.

Hemolysis rate of macDNA-Fe/PMCS was less than $1 \%$, which was well within the permissible limits of ISO 10993-4 standards, that is, macDNA-Fe/PMCS can be used for injections. 
a
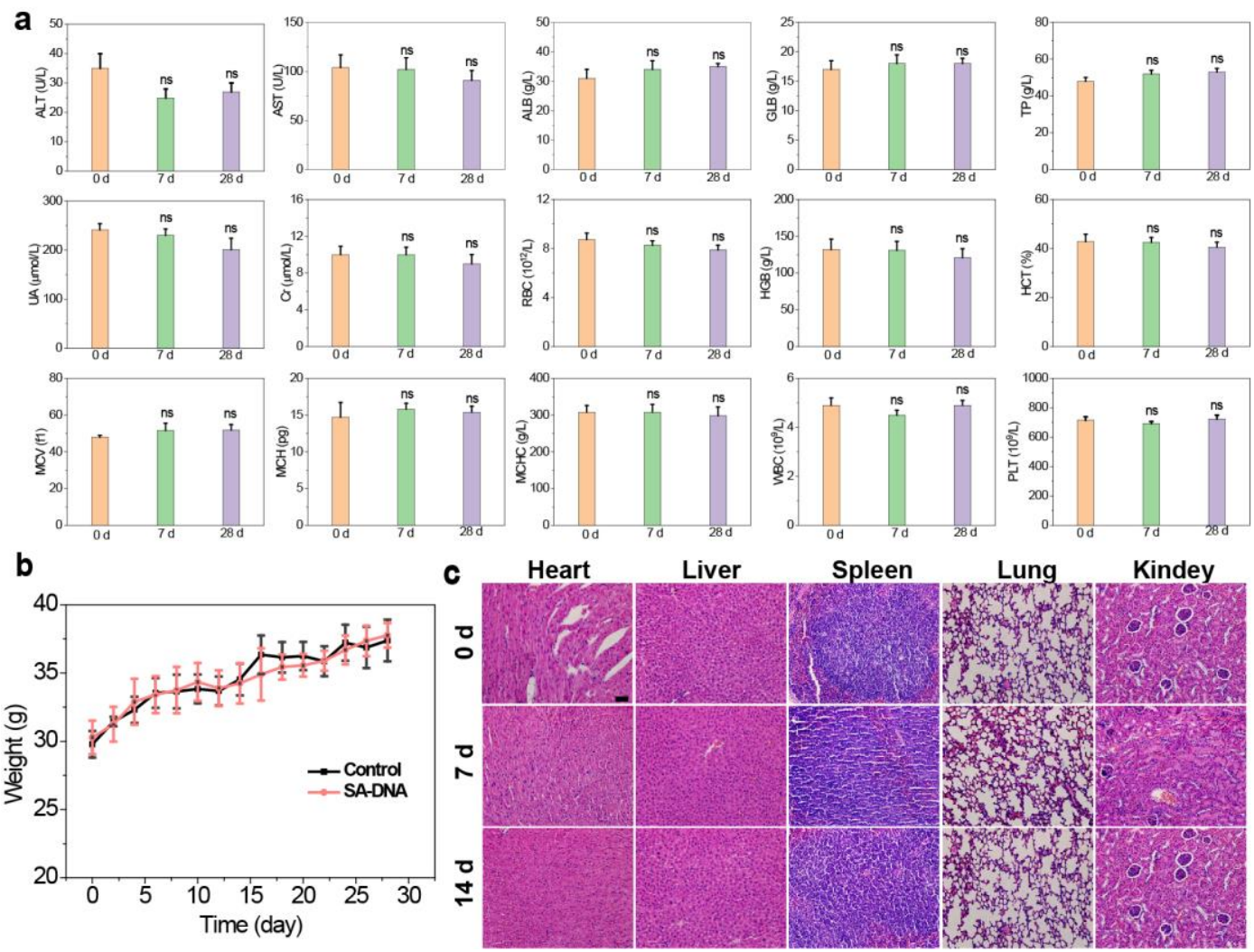

Figure 46. In vivo toxicity. a) Blood biochemical and haematological analysis of the healthy mice intravenously injected with macDNA-Fe/PMCS for 7 and 28 days. b) Body weights of mice treated with the macDNA-Fe/PMCS, record every two days. c) H\&E images obtained from the major organs of the macDNA-Fe/PMCS-treated mice for 7 and 28 days. Scale bars are $100 \mu \mathrm{m} . \mathrm{ns}$, no significance, $* P<0.05$, $* * P<0.01$ and $* * * P<0.001$ versus $0 \mathrm{~d}$. Data were presented as mean \pm s.d. $(n=5)$.

The hemolytic test and the standard haemotology assay as well as hematoxylin and eosin (H\&E) staining assay of major organs elucidated the high histocompatibility of macDNA-Fe/PMCS. Their influence on liver function was revealed by alanine transaminase (ALT), aspartate transaminase (AST), alburnin (ALB), globulin (GLB), and total protein (TP), while the kidney status was reflected by blood urea nitrogen (BUN) and creatinine (CREA) markers. There were no distinct differences between macDNA-Fe/PMCS treated group and control group, suggesting that macDNA-Fe/PMCS had negligible toxicity to liver and kidney. During the whole evaluation period, the mice growth was also hardly affected by macDNA-Fe/PMCS, further demonstrating the biocompatibility of macDNA-Fe/PMCS. Meanwhile, the hematoxylin and eosin (H\&E) staining of major organs elucidated the high 
histocompatibility of macDNA-Fe/PMCS. Collectively, these results validated the biosafety of macDNA-Fe/PMCS. 


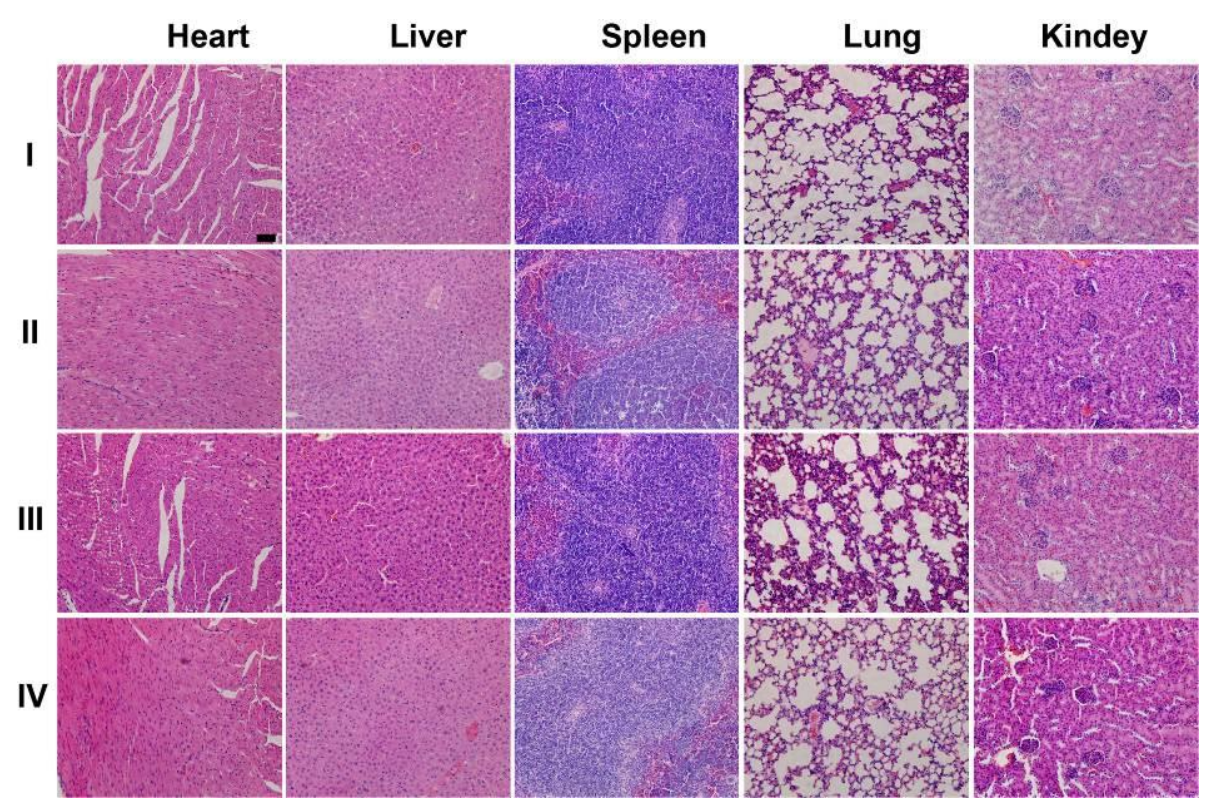

Figure 47. Histopathological examinations of the mice with breast cancer via H\&E staining of major organs after 14 days of treatment with various materials. I: Control, II: $\mathrm{Fe}_{3} \mathrm{O}_{4}$, III: macDNA-Fe/PMCS+liproxstatin-1, IV: macDNA-Fe/PMCS. Scale bars are $100 \mu \mathrm{m}$. 


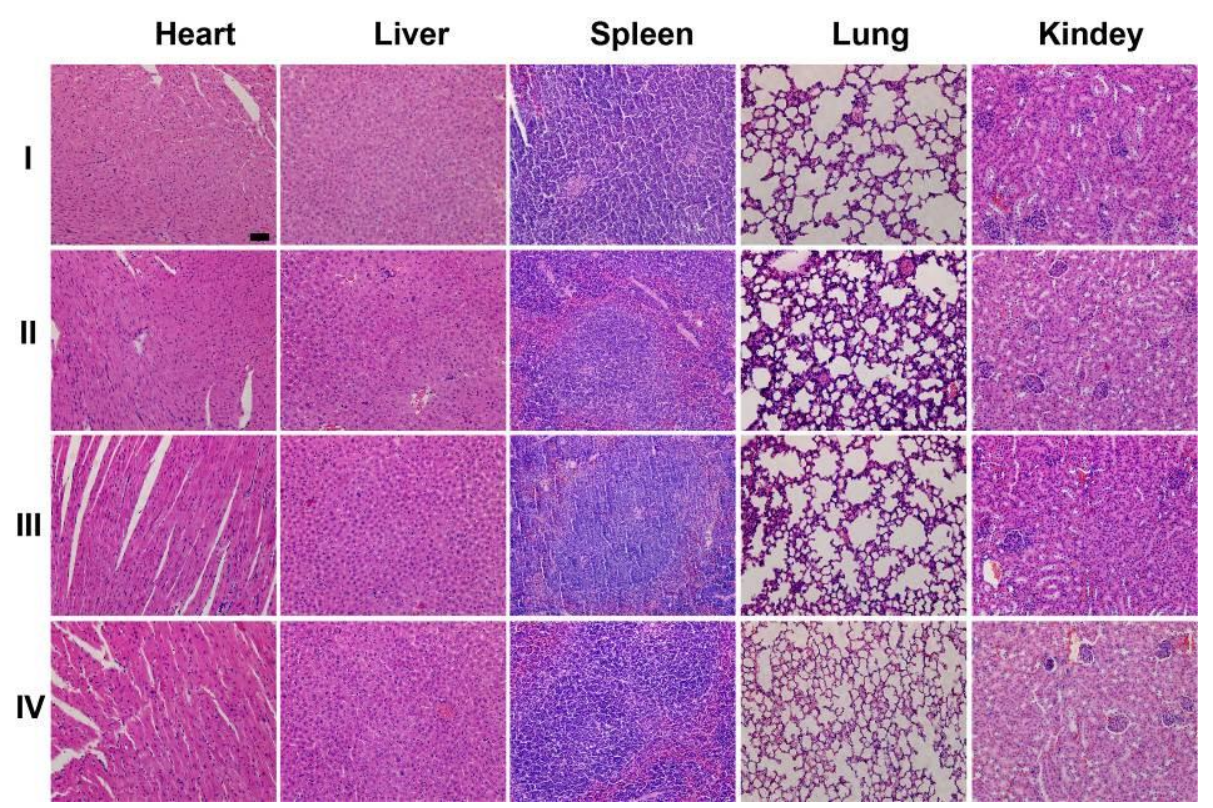

Figure 48. Histopathological examinations of the mice with colon cancer via H\&E staining of major organs after 14 days of treatment with various materials. I: Control, II: $\mathrm{Fe}_{3} \mathrm{O}_{4}$, III: macDNA-Fe/PMCS+liproxstatin-1, IV: macDNA-Fe/PMCS. Scale bars are $100 \mu \mathrm{m}$. 
Table 1. EXAFS fitting parameters at the Fe K-edge for Fe/PMCS.

\begin{tabular}{ccccccc}
\hline Sample & Shell & $N^{a}$ & $R(\AA)^{b}$ & $\sigma^{2}\left(\AA^{2} \cdot 10^{3}\right)^{c}$ & $\Delta E 0(\mathrm{eV})^{d}$ & $R$ factor (\%) \\
\hline Fe/PMCS & Fe-N & 4.1 & 1.94 & 8.5 & -3.2 & 0.7 \\
\hline
\end{tabular}

${ }^{a} N$ : coordination numbers; ${ }^{b} R$ : bond distance; ${ }^{c} \sigma^{2}$ : Debye-Waller factors; ${ }^{d} \Delta E_{0}$ : the inner potential correction. $R$ factor: goodness of fit. $S_{0}^{2}, 0.8$, was obtained from the experimental EXAFS fit of $\mathrm{Fe}_{2} \mathrm{O}_{3}$ reference by fixing PMCS as the known crystallographic value and was fixed to all the samples.

Error bounds that characterize the structural parameters obtained by EXAFS spectroscopy were estimated as $\mathrm{N} \pm 20 \% ; \mathrm{R} \pm 1 \% ; \sigma^{2} \pm 20 \% ; \Delta \mathrm{E} 0 \pm 20$. 
1. Kresse, G.; Furthmüller, J., Efficiency of ab-Initio Total Energy Calculations for Metals and Semiconductors Using a Plane-Wave Basis Set. Comput. Mater. Sci. 1996, 6 (1), 15-50.

2. Kresse, G.; Joubert, D., From Ultrasoft Pseudopotentials to the Projector Augmented-Wave Method. Phys. Rev. B 1999, 59 (3), 1758-1775.

3. Ernzerhof, M.; Scuseria, G. E., Assessment of the Perdew-Burke-Ernzerhof Exchange-Correlation Functional. J. Chem. Phys. 1999, 110 (11), 5029-5036.

4. Perdew, J. P.; Burke, K.; Ernzerhof, M., Generalized Gradient Approximation Made Simple. Phys. Rev. Lett. 1996, 77 (18), 3865-3868.

5. Grimme, S.; Antony, J.; Ehrlich, S.; Krieg, H., A Consistent and Accurate $a b$ Initio Parametrization of Density Functional Dispersion Correction (DFT-D) for the 94 Elements H-Pu. J. Chem. Phys. 2010, 132 (15), 154104.

6. Yang, K.; Dai, Y.; Huang, B.; Feng, Y. P., Density-Functional Characterization of Antiferromagnetism in Oxygen-Deficient Anatase and Rutile $\mathrm{TiO}_{2}$. Phys. Rev. B 2010, 81 (3), 033202.

7. Arroyo-de Dompablo, M. E.; Morales-García, A.; Taravillo, M., DFT+U Calculations of Crystal Lattice, Electronic Structure, and Phase Stability under Pressure of $\mathrm{TiO}_{2}$ Polymorphs. J. Chem. Phys. 2011, 135 (5), 054503.

8. Grimme, S.; Ehrlich, S.; Goerigk, L., Effect of the Damping Function in Dispersion Corrected Density Functional Theory. J. Comput. Chem. 2011, 32 (7), 1456-1465.

9. Ji, S.; Jiang, B.; Hao, H.; Chen, Y.; Dong, J.; Mao, Y.; Zhang, Z.; Gao, R.; Chen, W.; Zhang, R.; Liang, Q.; Li, H.; Liu, S.; Wang, Y.; Zhang, Q.; Gu, L.; Duan, D.; Liang, M.; Wang, D.; Yan, X. et al. Matching the Kinetics of Natural Enzymes with a Single-Atom Iron Nanozyme. Nat. Catal. 2021,4 (5), 407-417.

10. Huo, M.; Wang, L.; Wang, Y.; Chen, Y.; Shi, J., Nanocatalytic Tumor Therapy by Single-Atom Catalysts. ACS Nano 2019, 13 (2), 2643-2653.

11. Zhao, C.; Xiong, C.; Liu, X.; Qiao, M.; Li, Z.; Yuan, T.; Wang, J.; Qu, Y.; Wang, X.; Zhou, F.; Xu, Q.; Wang, S.; Chen, M.; Wang, W.; Li, Y.; Yao, T.; Wu, Y.; Li, Y., Unraveling the Enzyme-Like Activity of Heterogeneous Single Atom Catalyst. Chem. Commun. 2019, 55 (16), 2285-2288. 
12. Gao, L.; Zhuang, J.; Nie, L.; Zhang, J.; Zhang, Y.; Gu, N.; Wang, T.; Feng, J.; Yang, D.; Perrett, S.; Yan, X., Intrinsic Peroxidase-Like Activity of Ferromagnetic Nanoparticles. Nat. Nanotech. 2007, 2 (9), 577-583.

13. Hu, Y.; Gao, X. J.; Zhu, Y.; Muhammad, F.; Tan, S.; Cao, W.; Lin, S.; Jin, Z.; Gao, X.; Wei, H., Nitrogen-Doped Carbon Nanomaterials as Highly Active and Specific Peroxidase Mimics. Chem. Mater. 2018, 30 (18), 6431-6439.

14. Zhang, W.; Hu, S.; Yin, J. J.; He, W.; Lu, W.; Ma, M.; Gu, N.; Zhang, Y., Prussian Blue Nanoparticles as Multienzyme Mimetics and Reactive Oxygen Species Scavengers. J. Am. Chem. Soc. 2016, 138 (18), 5860-5865. 